\title{
Interactions between pulmonary hemodynamics and lung mechanics
}

$\mathrm{PhD}$ Thesis

Gergely Albu MD

Department of Medical Physics and Informatics

University of Szeged, Hungary 


\section{Table of Contents}

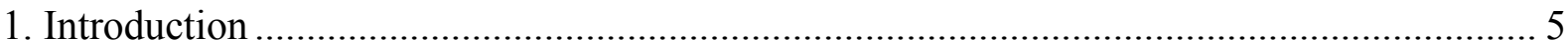

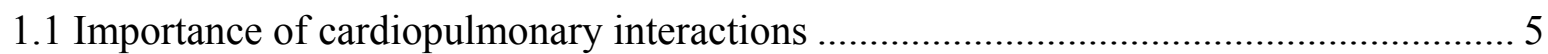

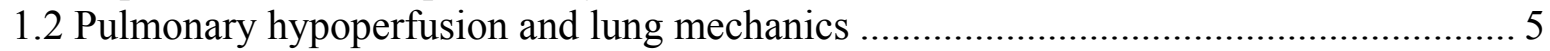

1.3 Role of transpulmonary pressure in the pulmonary mechanical and vascular changes ... 6

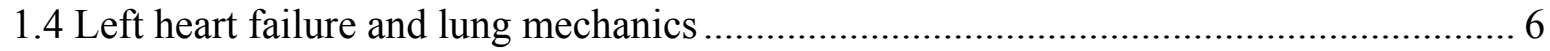

1.5 Effect of extracorporeal circulation on postoperative respiratory function .................... 7

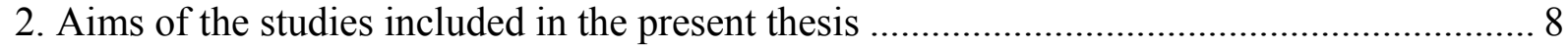

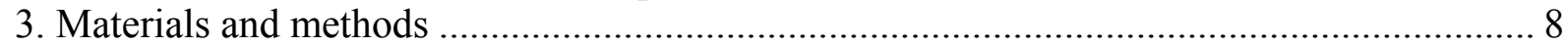

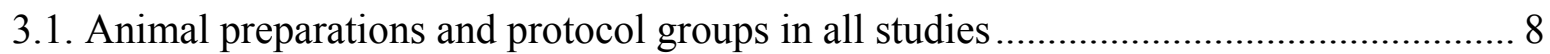

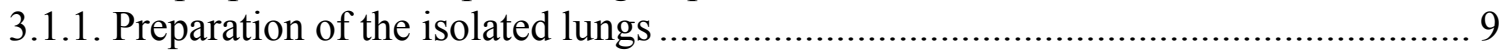

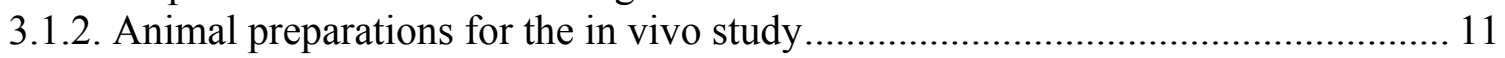

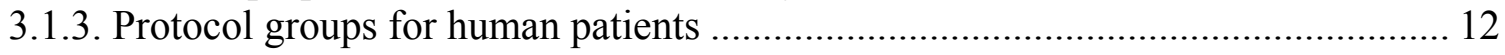

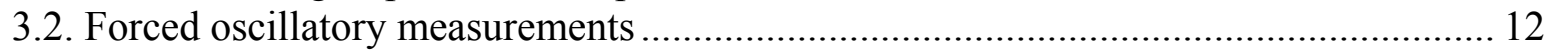

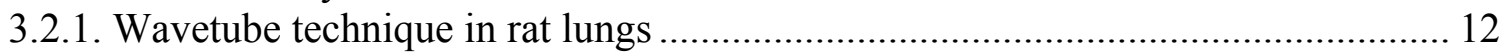

3.2.2. Tracking measurements for continuous assessment of the lung mechanics .......... 13

3.2.3. Impedance measurements with the classical setup in patients ............................. 14

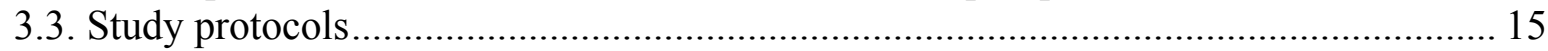

3.3.1. Effects of pulmonary capillary pressure on lung mechanics................................ 15

3.3.2. Lung mechanical and vascular changes during positive- and negative-pressure lung

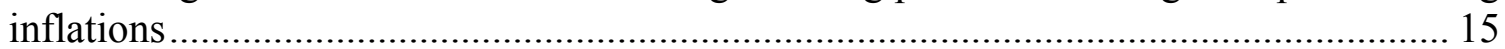

3.3.2. Brochial hyper-responsiveness after coronary ischemia ................................... 16

3.3.4. Changes in airway and respiratory tissue mechanics after cardiac surgery ........... 17

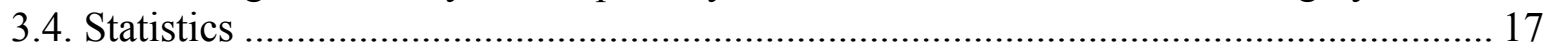

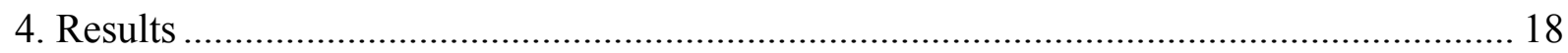

4.1. The influence of the pulmonary microvascular pressure on the mechanical properties of

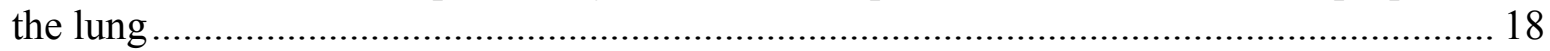

4.2. The effect of positive- and negative-pressure lung inflations on lung mechanics and the

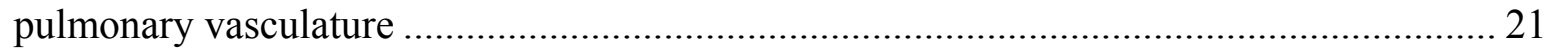

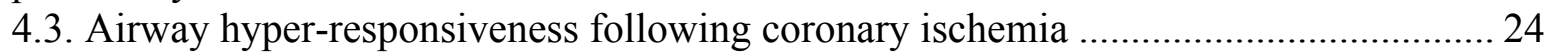

4.4. The effect of cardiac surgery on the mechanics of the respiratory system ................... 27

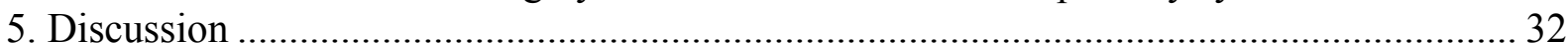

5.1. The contribution of the pulmonary microvascular pressure in the maintenance of the

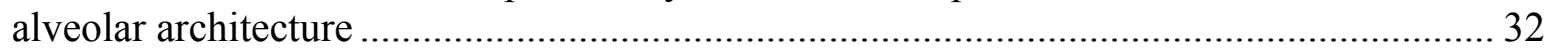

5.2. Mechanical and vascular changes in the lungs during inflation-defleation maneuvers

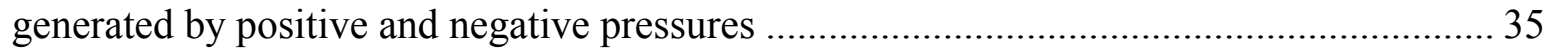

5.3. Mechanisms of bronchial hyper-responsiveness following coronary ischemia ............ 38

5.4. Changes in the mechanics of the respiratory system after cardiac surgery .................. 41

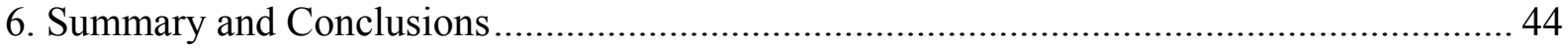

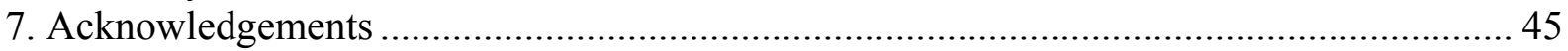

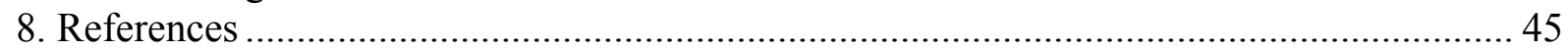




\section{List of papers included in this thesis}

I. Albu G, Habre W, Fontao F, Morel DR, Peták F. The contribution of the pulmonary microvascular pressure in the maintenance of an open lung during mechanical ventilation. Respir Physiol Neurobiol. 2007 Aug 1;157(2-3):262-9.

II. Albu G, Peták F, Fontao F, Biton C, Pache JC, Habre W. Mechanisms of airway hyperresponsiveness after coronary ischemia. Respir Physiol Neurobiol. 2008 Aug 31;162(3):176-83.

III. Peták F, Albu G, Lele E, Hantos Z, Morel DR, Fontao F, Habre W. Lung mechanical and vascular changes during positive- and negative-pressure lung inflations: importance of reference pressures in the pulmonary vasculature. J Appl Physiol. 2009 Mar;106(3):935-42.

IV. Albu G, Babik B, Késmárky K, Balázs M, Hantos Z, Peták F. Changes in airway and respiratory tissue mechanics after cardiac surgery. Ann Thorac Surg. 2010 Apr;89(4):1218-26.

\section{List of papers related to the subject of this thesis}

I. Habre W, Petak F, Ruchonnet-Metrailler I, Donati Y, Tolsa JF, Lele E, Albu G, Beghetti M, Barazzone-Argiroffo C. The role of endothelin-1 in hyperoxia-induced lung injury in mice. Respir Res. 2006 Mar 27;7:45.

II. Thamrin C, Albu G, Sly PD, Hantos Z. Negative impact of the noseclip on high-frequency respiratory impedance measurements. Respir Physiol Neurobiol. 2009 Jan 1;165(1):115-8

III. Habre W, Janosi TZ, Fontao F, Meyers C, Albu G, Pache JC, Petak F. Mechanisms for lung function impairment and airway hyperresponsiveness following chronic hypoxia in rats. Am J Physiol Lung Cell Mol Physiol. 2010 Apr;298(4):L607-14.

\section{Figures and tables}

Table 1. Anthropometric data of the patients having cardia surgery

Figure 1. Set-up for isolated lungs

Figure 2. Changes in the mechanical properties of the lung at different pulmonary capillary pressures and PEEP levels

Figure 3. Relative changes in the airway and lung tissue parameters at different pulmonary capillary pressures and PEEP levels

Figure 4. Pressure-volume curves in a representative isolated perfused lung 
Figure 5. Relationships between the indices extracted from the $\mathrm{P}-\mathrm{V}$ curves

Figure 6. Changes in pulmonary vascular resistance and in the mechanical parameters of the lung during positive and negative pressure inflation-deflation maneuvers

Figure 7. Changes in the airway and parenchymal mechanical parameters as functions of the transpulmonary pressure

Figure 8. Changes in vascular resistance as a function of the transpulmonary pressure and lung volume

Figure 9. Lung mechanical parameters and vascular resistance at different transpulmonary pressures during positive and negative pressure lung inflations

Figure 10. Changes in the mechanical properties of the respiratory system following iv. boluses of serotonin before and after coronary ischemia

Figure 11. Relative changes in the mechanical parameters of the respiratory system at the peak response to serotonin boluses in the control and ischemia group

Figure 12. Relationship between end-diastolic left-ventricular pressure and the airway responsiveness to 20 and $30 \mu \mathrm{g} / \mathrm{kg}$ serotonin in the ischemia group

Figure 13. Lung histological sections obtained from a representative control rat and one with myocardial ischemia

Figure 14. Histological findings in the control rats and in the rats with myocardial ischemia

Figure 15. Real and imaginary parts of the respiratory input impedance in a representative patient before and after cardiac surgery

Figure 16. Parameters obtained from the respiratory input impedance before and after cardiac surgery for the overall study population

Figure 17. Mean values of the airway and respiratory tissue parameters before and after cardiac surgery in two groups of patients

Figure 18. Changes in airway resistance and respiratory elastance during the first postoperative week relative to the preoperative level

Figure 19. Mean values of the respiratory system parameters before and after cardiac surgery in patients with or without obesity

Figure 20. Changes in airway resistance and respiratory elastance during the first postoperative week relative to the preoperative level in cardiac patients with or without obesity 


\section{Glossary of terms}

$\alpha \quad=$ Exponent of the angular frequency in the constant-phase tissue model

$\alpha$-SMA $=$ alpha smooth muscle actin

$\gamma=$ Complex propagation wavenumber of the wavetube

$\omega=$ Angular frequency

$\eta \quad=$ Tissue hysteresivity

$\mathrm{BHR}=$ Bronchial hyperresponsiveness

$\mathrm{Cg}=$ Alveolar gas compliance

$\mathrm{CPB}=$ Cardiopulmonary bypass

$\mathrm{E}=$ Elastance

EDLVP $=$ End dyastolic left ventricular pressure

FOT $=$ Forced oscillation technique

$\mathrm{G}=$ Constant-phase tissue damping

$\mathrm{H}=$ Constant-phase tissue elastance

Iaw = Airway inertance

ID $=$ Internal diameter

ip $\quad=$ Intraperitoneal

iv $\quad=$ Intravenous

$\mathrm{j} \quad=$ Imaginary unit

$\mathrm{L}=$ Length of the wave tube

MI = Myocardial ischemia

NPLI = Negative pressure lung inflation

OPCAB = Off-pump coronary artery bypass

$\mathrm{P}_{1}=$ Pressure at the loudspeaker end of the wave tube

$\mathrm{P}_{2} \quad=$ Pressure at the distal end of the wave tube

Pao = Airway opening pressure

Palv = Alveolar pressure

Paw = Airway pressure

Pc = Pulmonary capillary pressure

$\mathrm{Pc}_{\mathrm{EST}}=$ Estimated pulmonary capillary pressure

$\mathrm{PCO}_{2}=$ fractional pressure of carbon dioxide

PEEP $=$ Positive end-expiratory pressure

Pla = Left atrial pressure

$\mathrm{PO}_{2}=$ fractional pressure of oxygen

Ppa = Pulmonary arterial pressure

PPLI = Positive pressure lung inflation

Ptp = Transpulmonary pressure

$\mathrm{P}-\mathrm{V}=$ Pressure-volume relationship

$\mathrm{Qp} \quad=$ Pulmonary blood flow

Raw = Airway resistance

$\mathrm{RL}=$ Pulmonary resistance

Rrs $\quad=$ Real part of impedance

$\mathrm{Rv}=$ Pulmonary vascular resistance

$\mathrm{SE}=$ Standard error

$\mathrm{V}^{\prime}=$ Air flow

VT $=$ Tidal volume

Xrs = imaginary part of impedance

$\mathrm{Z}_{0} \quad=$ Characteristic impedance of the wavetube

$\mathrm{Z}_{\text {in }} \quad=$ Input impedance

$\mathrm{ZL}=$ Pulmonary input impedance

$\mathrm{Zrs}=$ Impedance of the respiratory system 


\section{Introduction}

\subsection{Importance of cardiopulmonary interactions}

The pulmonary system maintains gas exchange by the continuous control of elements assuring the transfer of the respiratory gases and the blood in the pulmonary vasculature. This unity of conducting airways terminating in the alveoli and the pulmonary capillaries forms a complex system where any change in one compartment affect the other via cardiopulmonary interactions. This mechanical interdependence is manifested in airway and/or lung tissue changes subsequent to acute or chronic alterations in the pulmonary hemodynamics $[1,2]$. Understanding the relationships between the pulmonary vasculature and bronchoalveolar networks has major importance in pathophysiological conditions encountered in various cardiopulmonary diseases, such as coronary ischaemia leading to left heart failure [3-5], congenital heart malfunctions $[6,7]$ or valvular disfunction $[5,8]$.

\subsection{Pulmonary hypoperfusion and lung mechanics}

There is increasing evidence that not only lung congestion, but also hypoperfusion in the pulmonary circulation causes impairments in lung mechanics [1, 2, 9-11]. It has been also demonstrated that, at low vascular pressure, the lung mechanics is compromised by loss of the mechanical tethering effect exerted by the pressurized pulmonary capillary network [2]. These results led to the conclusion that physiological pressure in the pulmonary capillaries is an important mechanical factor for the maintenance of the stability of the alveolar architecture. The loss of pressurized pulmonary capillaries and the subsequent breakdown in the maintenance of the optimal alveolar geometry may enhance the development of atelactasis. Various clinical conditions, such as embolism, hypovolemia and cardiopulmonary bypass, are associated with hypoperfusion of the pulmonary system. This phenomenon has been shown to be of greater importance in situations when the lung volume is lowered to below the closing volume [2]. 


\subsection{Role of transpulmonary pressure in the pulmonary mechanical and vascular changes}

Changes in lung volume result in alterations in both the complex structure of the conducting airways embedded in the lung parenchyma and the pulmonary vasculature. Positive-pressure lung inflation (PPLI), commonly applied to maintain gas exchange during mechanical ventilation, is far from being physiological and results in a number of side-effects, originating from the high positive intrathoracic pressures that may subsequently worsen pre-existing pulmonary lesions. PPLI may jeopardize the pulmonary hemodynamic conditions, mainly via compression of the pulmonary vessels [12-17]. These adverse pulmonary hemodynamic effects do not exist during normal breathing, since negative-pressure lung inflation (NPLI) generated by a negative pressure around the lungs exerts a different pressure gradient across the pulmonary vascular wall that generates a radial traction on the vessels as the lung parenchyma expands [18]. There is a consensus in the literature that PPLI decreases the airway resistance (Raw), alters the viscoelastic properties of the lungs [19-21] and increases the pulmonary vascular resistance $(\mathrm{Rv})[12,13,15-18,22]$. However, these previous studies addressed either the pulmonary vascular or the lung mechanical changes, their simultaneous alterations have not been characterized. In contrast with the consistent data obtained for PPLI, the findings on pulmonary hemodynamic changes during NPLI are still controversial $[13,15$, $18,23]$ mainly due to the inconsistency in the measurement of pulmonary vascular pressures. Whereas one study demonstrated a monotonic decrease in Rv during NPLI [18] others have documented a mild drop in $\mathrm{Rv}$, followed by gradual increases at high lung volumes [13, 15, 23]. All these previous studies characterized the pulmonary vascular and lung mechanical changes under steady-state conditions, the mechanical changes in these compartments during dynamic inflation-deflation maneuvers remain unknown.

\subsection{Left heart failure and lung mechanics}

Previous reports led to conflicting conclusions about the lung mechanical effects following left heart failure and the subsequent alterations in lung responsiveness to exogenous constrictor stimuli. Some authors demonstrated the induction of bronchial hyperresponsiveness (BHR) to lung provocations [3-5, 8] whereas others found no evidence of BHR in patients with chronic congestive heart failure $[24,25]$. In addition to this 
controversy, the underlying pathophysiological mechanisms leading to airway narrowing and occasionally BHR after pulmonary lung congestion have not been completely clarified.

A decrease in the airway cross-sectional area due to compression of the tracheobronchial tree by the dilated pulmonary vessels has been reported to be a major contributor to BHR [3, 8], as have thickened bronchial walls [26] and peribronchial edema [27, 28]. However, a reduction of the left ventricular preload by diuretics, leading to a partial clearance of the edema fluid, had no effects on the baseline lung mechanics and responsiveness [4], suggesting that the pulmonary consequences of left heart failure cannot be fully explained on a geometric basis or by fluid accumulation around the airways. Besides these mechanisms, the development of interstitial edema may uncouple the airways from the lung parenchyma by altering the mechanical interdependence between the airways and the surrounding lung tissue [29]. The resulting loss of lung recoil may further potentiate the responsiveness of the airway smooth muscles to a constrictor stimulus.

\subsection{Effect of extracorporeal circulation on postoperative respiratory function}

Besides the lung functional changes observed following acute or chronic changes in pulmonary hemodynamics, the airway and respiratory tissue properties exhibit alterations in the postoperative period after heart surgeries. Forced spirometric measurements, which are feasible after complete healing of the chest wound, have revealed the long-term consequences of heart operations [30, 31]. Through examinations of gas-exchange parameters and inflammatory markers in the blood $[32,33]$, chest imaging $[32,34]$ and measurements of lung volumes [35] or extravascular lung water [36], pulmonary complications have been demonstrated to persist for about a week following the surgery, with the most severe respiratory symptoms observed around the second day postoperatively $[33,35]$. In spite of the ample evidence indicating the major importance of the first postoperative week in the manifestation of respiratory morbidity after cardiac surgery, there has essentially been no systematic lung function measurements aimed at characterizing the changes in the airway and respiratory tissue mechanics in this critical period. Application of the cardiopulmonary bypass (CPB) during surgery with subsequent ischemia and reperfusion in the pulmonary circulation may have particular role in the postoperative changes in lung function, however this adverse effect has not been systematically investigated. 


\section{Aims of the studies included in the present thesis}

The primary aim of the present thesis is a better understanding of the cardiopulmonary interactions related to various clinical conditions. The studies included were designed

- to characterize how physiological pulmonary hemodynamics contributes to the maintenance of the normal levels of mechanical parameters related to the airways and the lung parenchyma. We also aimed to assess whether this factor has an importance in contributing to the protection against lung function deterioration during mechanical conditions. More specifically we designed experiments to characterize the alterations in lung mechanics that occur when the lungs are ventilated at normal and lowered transpulmonary pressures (Ptp), which advances derecruitment, at different levels of pulmonary capillary pressures $(\mathrm{Pc})$.

- to investigate the differences between the pulmonary mechanical and vascular effects of mechanical ventilation based on PPLI and NPLI. We assessed in these experiments whether NPLI improves ventilation-perfusion distribution by facilitating lung recruitment and preventing hemodynamic impairment.

- to study the effects of pulmonary vascular engorgement following left heart failure on the lung mechanics and the development of BHR. Combined pulmonary hemodynamic, respiratory mechanical measurements and morphometric evaluations were performed to clarify the underlying pathophysiological mechanisms.

- to establish the time course of the postoperative changes in the airway and respiratory tissue mechanics by measuring the mechanical properties of the respiratory system the day before surgery and one week thereafter. Since measurements were made in patients undergoing cardiac surgery with or without $\mathrm{CPB}$, a further aim was to characterize the mechanical consequences of $\mathrm{CPB}$ in the first postoperative week.

\section{Materials and methods}

\subsection{Animal preparations and protocol groups in all studies}

All of the animal protocols were approved by the Institutional Animal Care Committee of the Canton of Geneva in Switzerland. The study protocol involving human patients was approved by the Human Research Ethics Committee of Szeged University in Hungary, and informed consent was obtained from all subjects enrolled. 


\subsubsection{Preparation of the isolated lungs}

Adult male Sprague-Dawley rats weighing 360-390 g were anesthetized with isoflurane (3\% induction, $1.4 \%$ maintenance dose), then tracheotomized with a polyethylene cannula (14gauge, Braun, Melsungen, Germany) and mechanically ventilated with a constant volumecycled rodent ventilator (model 683, Harvard Apparatus Inc., South Natick, MA, USA) with a tidal volume of $7 \mathrm{ml} / \mathrm{kg}$ and a respiratory rate of $70-80 / \mathrm{min}$ while a positive end-expiratory pressure (PEEP) of $2.5 \mathrm{cmH}_{2} \mathrm{O}$ was maintained. The airway opening pressure was monitored continuously (DP 45 transducer, 2D15 carrier demodulator, Validyne, Northridge, CA, USA).

The femoral artery was cannulated with a 28-gauge catheter (Portex, Hythe, GB) for monitoring the systemic blood pressure (model 156 PCE 06-GW2, Honeywell, Zurich, Switzerland). The femoral vein was also cannulated for drug delivery. Heparin (1.5 IU/g) was then administered i.v. for complete anticoagulation of the blood. Thirty-five millilitre of arterial blood was next gently withdrawn and it was continuously replaced by the same amount of i.v. infusion of colloid solution (hydroxyethyl-starch 6\%). This maneuver maintained a constant intravascular volume and a mean systemic blood pressure above 50 $\mathrm{mmHg}$, and minimized the risk of ischemic lesions in the lungs. The collected blood was centrifuged (4000 rpm for $10 \mathrm{~min}$ ) and $17 \mathrm{ml}$ of plasma was extracted. The resulting concentrated blood with a hematocrit level of $\sim 35 \%$ served as perfusate. The chest was widely retracted following a midline sternotomy, and a polyethylene catheter (14-gauge, Braun) was placed into the main pulmonary artery via the right ventricular outflow track, and connected to silicone tubing (1.47mm ID, Ulrich, St. Gallen, Switzerland). The animals were then completely exsanguinated by widely opening the left ventricle and the left atrium.

To minimize the warm ischemic time period until reperfusion, the lungs were immediately flushed via the pulmonary artery cannula with $30 \mathrm{ml}$ of cold $\left(10^{\circ} \mathrm{C}\right)$ hydroxyethyl-starch $6 \%$ solution from a height of $30 \mathrm{~cm}$. Through the left ventriculotomy, another catheter was placed into the left ventricle, into which a Combifix ${ }^{\circledR}$-Adapter (Braun) was tightly fixed and connected to medical grade silicone tubing. Finally, a third catheter (polyethylene tubing, ID $0.88 \mathrm{~mm}$, Portex, Hythe, GB) was introduced directly into the left atrium for measurement of the left atrial pressure (Pla). The surgical preparations were performed in a sterile manner. The lungs and the heart were excised in a single block, dissected free of adjacent tissue. Then the heart-lung block was placed in a thermostabilized, humidified Plexiglass chamber and it was suspended from an isometric force displacement transducer (Grass FT03, Quincy, MA, USA), to continuously measure weight changes. 
Establishment of the perfusion of the isolated rat lungs. The experimental set-up is shown schematically in Figure 1. The lungs were ventilated by applying positive pressure in the trachea by using the rodent ventilator with room air mixed with $5 \% \mathrm{CO}_{2}$ at a respiratory rate of 50/min, a tidal volume (VT) of $7 \mathrm{ml} / \mathrm{kg}$ and a PEEP of $2.5 \mathrm{cmH}_{2} \mathrm{O}$. Peak airway pressure (Paw) was measured with a pressure transducer (model Z46169, Gould). VT and airflow (V') were measured with a screen pneumotachograph (type 17212, Gould Godart, Bilthoven, The Netherlands). The perfusion circuit was primed with the rat's own blood after filtration.

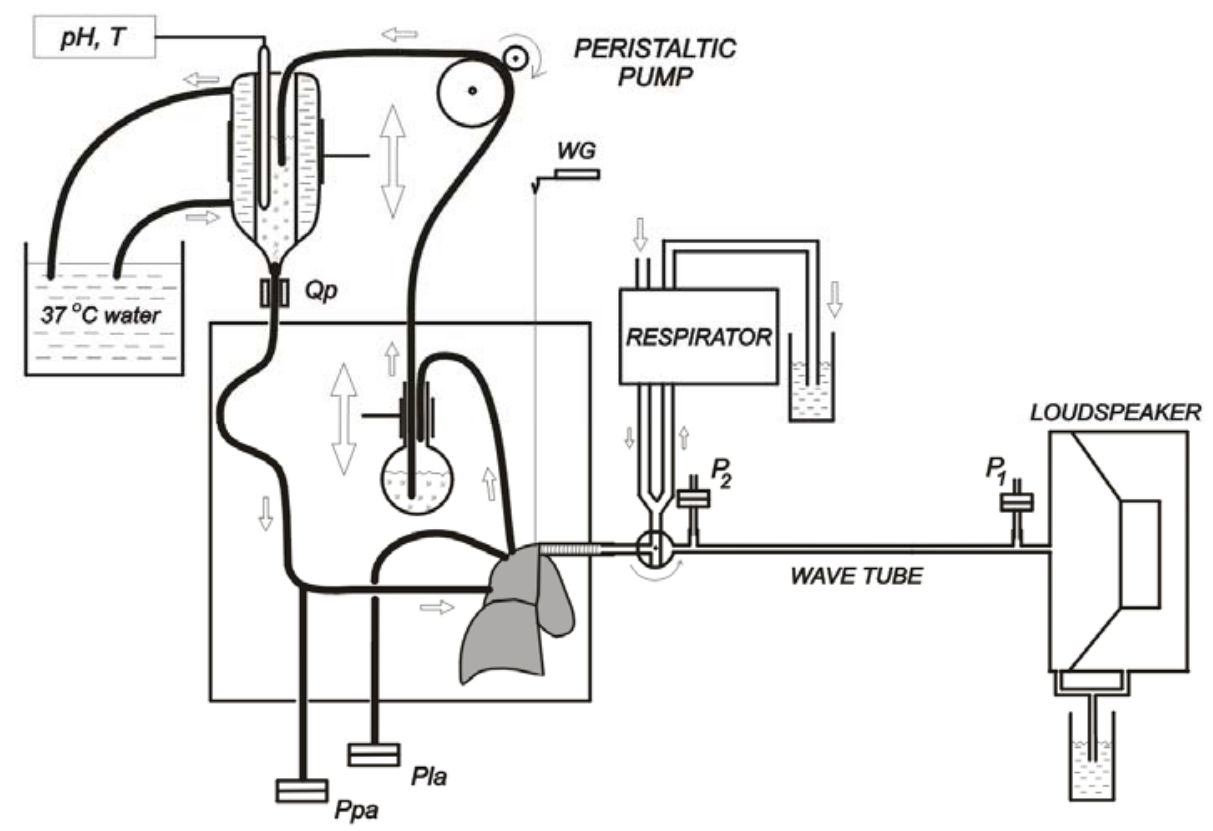

Figure 1. Experimental set-up for isolated rat lungs

Lung perfusion was performed from a perfusion reservoir set initially to have a pulmonary artery perfusion pressure (Ppa) of $15 \mathrm{mmHg}$. The distal extremity of the left ventricular outflow cannula was positioned at a height to obtain a left atrial pressure (Pla) of $5 \mathrm{mmHg}$ at the start of the reperfusion, which produced a pulmonary capillary pressure (Pc) of $10 \mathrm{mmHg}$ and West zone 3 conditions (Ppa>Pla>mean Paw). The blood dripping from this cannula was collected in a cylinder, and it was aspirated from this reservoir with polyethylene tubing passing through a roller pump (Ismatec Pump, Glattburg, Zurich, Switzerland). A transit-time flowmeter (T-201 CDS, Transonic Systems, Ithaca, NY) was placed between the perfusion reservoir and the catheter cannulating the main pulmonary artery for continuous monitoring of the blood flow (Qp). Ppa and Pla were measured continuously with calibrated pressure transducers (model 156-PC 06-GW2, Honeywell, Zurich, Switzerland) zeroed at the level of the lung hilum. Vascular resistance $(\mathrm{Rv})$ was calculated from the vascular pressure and flow signals as $\mathrm{Rv}=(\mathrm{Ppa}-\mathrm{Pla}) / \mathrm{Qp}$. Pc was estimated $\left(\mathrm{Pc}_{\mathrm{EST}}\right)$ by applying the Gaar 
equation $\left[\mathrm{Pc}_{\mathrm{EST}}=\mathrm{Pla}+0.44 \times(\mathrm{Ppa}-\mathrm{Pla})\right][37]$ and was used to assess the capillary filling pressure before the maneuvers. V', VT, pressures, circuit flow, and the calculated Rv were recorded and stored at a sampling rate of $50 \mathrm{~Hz}$ via an analog-to-digital interface converter (Biopac, Santa Barbara, CA) on a microcomputer (AST, Limerick, Ireland). The temperature and $\mathrm{pH}$ of the perfusate were measured with a $\mathrm{pH}$-meter (691 $\mathrm{pH}$-meter, Metrohm, Herisau, Switzerland). The $\mathrm{pH}$ was maintained between 7.35 and 7.45 and was corrected with sodium bicarbonate or a change of the inspired $\mathrm{CO}_{2}$ if this was indicated by the blood gas analysis (model 505, Acid Base Laboratory, Copenhagen, Denmark). Steady-state gas exchange was confirmed by the stable $\mathrm{PO}_{2}, \mathrm{PCO}_{2}$, and haematocrit levels during the experiments.

\subsubsection{Animal preparations for the in vivo study}

For the in vivo experiments adult male Sprague-Dawley rats (weighing 342-395 g) were anaesthetized by intraperitoneal injection of pentobarbital $(70-90 \mathrm{mg} / \mathrm{kg}$ of a $50 \mathrm{mg} / \mathrm{ml}$ solution) and placed in supine position. The rats were then intubated with a $14 \mathrm{G}$ polyethylene cannula and were mechanically ventilated with a tidal volume of $7 \mathrm{ml} / \mathrm{kg}$ bodyweight, a positive end-expiratory pressure of $2.5 \mathrm{cmH}_{2} \mathrm{O}$, and a respiratory rate of $70-80 / \mathrm{min}$. Anesthesia was maintained with pentobarbital administered iv. every $40 \mathrm{~min}(5 \mathrm{mg} / \mathrm{kg})$. After the femoral artery and vein were cannulated (Abocath $22 \mathrm{G}$ ), the arterial line was used for continuous blood pressure monitoring and blood gas analysis, the femoral vein was used for drug delivery. After the blood gas analysis the parameters of the mechanical ventilation were adjusted to maintain normal gas exchange if it was necessary. The airway pressure, ECG and rectal temperature were continuously monitored by the data collection and acquisition system (Biopac). Fentanyl was administered intravenously $(15 \mu \mathrm{g} / \mathrm{kg})$ to ensure adequate analgesia. The heart in each rat was then surgically exposed by a left-lateral thoracotomy (5th intercostal). A prophylactic dose of lidocaine ( $2 \mathrm{mg} / \mathrm{kg}$ intravenously) was administered to prevent arrhythmias that may arise from ligation of the coronary artery. After opening of the pericardium, a silk thread was sutured around the proximal part of the descending interventricular coronary artery (next to the departure of the pulmonary artery). After that the animals were randomly assigned to two different protocol groups. In the ischemia group [Group I $(\mathrm{n}=9)$ ], the knot was tightened to induce myocardial ischemia, while in the rats enrolled in the control group [Group $C(n=6)$ ], the thread was removed without occlusion of the coronary artery. After removing the residual air in the pleural cavity the thorax was closed and a long-acting local anesthetic (bupivacaine $0.25 \%, 0.8-1.2 \mathrm{ml}$ ) was infiltrated around the 
surgical wounds. $0.5 \mathrm{mg} / \mathrm{kg}$ of buprenorphine (Temgesic) was injected subcutaneously before the rat was allowed to recover from the anesthesia, and this dose was repeated $18 \mathrm{~h}$ later to ensure adequate analgesia. Four weeks later, the animals were anaesthetized and intubated again as detailed above, and the second part of the study was accomplished.

\subsubsection{Protocol groups for human patients}

16 patients scheduled for elective valve replacement requiring cardiopulmonary bypass (CPB) with or without coronary bypass graft and another 19 patients in whom off-pump coronary artery bypass (OPCAB) surgery was performed were involved in the study protocol. Table 1 presents the anthropometric data of the patients.

\begin{tabular}{lcccc}
\hline & \multicolumn{2}{c}{ CPB $(\mathrm{n}=16)$} & \multicolumn{2}{c}{ OPCAB $(\mathrm{n}=19)$} \\
\hline Age (years) & $67 \pm 2.5$ & $(46-80)$ & $62 \pm 1.5$ & $(52-74)$ \\
Weight $(\mathrm{kg})$ & $76 \pm 3$ & $(58-101)$ & $82 \pm 4.2$ & $(57-133)$ \\
Height $(\mathrm{cm})$ & $163 \pm 2.5$ & $(149-188)$ & $166 \pm 2.1$ & $(145-177)$ \\
BMI & $29 \pm 1.2$ & $(21.6-37.6)$ & $29.5 \pm 1.3$ & $(21.7-46)$ \\
Sex (M/F) & $6 / 10$ & & $15 / 4$ & \\
\hline
\end{tabular}

Table 1. Values are mean \pm standard error; (minimum-maximum). BMI = body mass index

Anesthesia and surgery. After premedication $(0.07 \mathrm{mg} / \mathrm{kg}$ morphine and $0.07 \mathrm{mg} / \mathrm{kg}$ midazolam), iv anesthesia $(0.03 \mathrm{mg} / \mathrm{kg}$ midazolam, $0.5-0.7 \mu \mathrm{g} / \mathrm{kg}$ sufentanil and $0.3 \mathrm{mg} / \mathrm{kg}$ propofol) and muscle relaxation $(0.6 \mathrm{mg} / \mathrm{kg}$ rocuronium) were maintained in all patients. Subsequent to orotracheal intubation, the patients were mechanically ventilated. A median sternotomy was performed in all patients. OPCAB surgery was performed by using the Octopus system, whereas in the CPB patients a membrane oxygenator was applied.

\subsection{Forced oscillatory measurements}

\subsubsection{Wavetube technique in rat lungs}

The contributions of the airway and tissue mechanical properties to the total lung or respiratory system resistance were estimated by the forced oscillation technique (FOT) by measuring the mechanical impedance of the isolated lungs (ZL) or the total respiratory system (Zrs) with closed chest, as described in detail previously [1, 38]. Briefly, the tracheal cannula was connected from the respirator to a loudspeaker-in-box system at end-expiration. The pressure in the loudspeaker box was set to the level of PEEP prior to the measurements to 
maintain a constant transpulmonary pressure level during the oscillatory recordings. The loudspeaker generated a small-amplitude pseudorandom oscillatory signal with frequency components between 0.5 and $21 \mathrm{~Hz}$ through a polyethylene wave-tube with known geometry. Two identical pressure transducers (model 33NA002D, ICSensors, Milpitas, CA, USA) were used to measure the lateral pressure at the loudspeaker end $\left(\mathrm{P}_{1}\right)$ and at the tracheal end $\left(\mathrm{P}_{2}\right)$ of the wave-tube. The input impedance $\left(Z_{\text {in }}\right)$ was calculated as the load impedance of the wavetube by using fast Fourier transformation with $4 \mathrm{~s}$ time windows and $95 \%$ overlapping to calculate the pressure transfer functions $\left(\mathrm{P}_{1} / \mathrm{P}_{2}\right)$ from $6 \mathrm{~s}$ long recordings [38].

$$
\left.\mathrm{Z}_{\text {in }}=\mathrm{Z}_{0} \sinh (\gamma \mathrm{L}) /\left[\mathrm{P}_{1} / \mathrm{P}_{2}\right)-\cosh (\gamma \mathrm{L})\right]
$$

where $\mathrm{L}$ is the length, $\mathrm{Z}_{0}$ is the characteristic impedance and $\gamma$ is the complex propagation wavenumber of the wavetube. The latter two parameters are determined by the geometrical data and the material constant of the tube wall and the air.

To separate the airway and tissue mechanics, a model containing a frequencyindependent airway resistance (Raw) and inertance (Iaw) in series with a constant-phase tissue model [39] including tissue damping $(\mathrm{G})$ and elastance $(\mathrm{H})$ was fitted to the impedance spectra by minimizing the differences between the measured and modeled impedance values:

$$
\mathrm{Z}_{(\mathrm{in})}=\mathrm{Raw}+\mathrm{j} \omega \mathrm{Iaw}+(\mathrm{G}-\mathrm{jH}) / \omega^{\alpha}
$$

where $\mathrm{j}$ is the imaginary unit, $\omega$ is the angular frequency $(2 \pi \mathrm{f})$, and $\alpha=2 / \pi \arctan (\mathrm{H} / \mathrm{G})$. Tissue hysteresivity was calculated as $\eta=\mathrm{G} / \mathrm{H}$ [40]. When this model is fitted to Zrs spectra, the parameter Raw primarily related to the overall airway geometry, as the contribution of the chest wall to the frequency independent Newtonian resistance is minor [41]. Similarly, the airways are dominated in the parameter Iaw [41].

The load impedance of the endotracheal tube and the connecting tubing was also determined, and the reported Raw and Iaw values were corrected by subtracting the instrumental resistance and inertance values.

\subsubsection{Tracking measurements for continuous assessment of the lung mechanics}

The respective contributions of the airway and tissue mechanical properties to the total lung resistance can be estimated by the modified FOT, which allows continuous assessment of ZL, as described in detail previously [20,42]. For the impedance recordings we used a small amplitude pseudorandom signal with frequency components between 0.5 and $19 \mathrm{~Hz}$ through a $100 \mathrm{~cm}$ long and $2 \mathrm{~mm}$ inner diameter polyethylene catheter serving as the wave tube. Lateral pressures $\left(\mathrm{P}_{1}, \mathrm{P}_{2}\right)$ were measured at both ends of the wavetube. The signals of $\mathrm{P}_{1}$ and $\mathrm{P}_{2}$ were low-pass filtered at $25 \mathrm{~Hz}$ and sampled with a microcomputer at a rate of $128 \mathrm{~Hz}$ and ZL was 
calculated as described previously. ZL recordings for the tracking measurements were $160 \mathrm{~s}$ long. The time window of the ZL calculation was $2 \mathrm{~s}$ and successive overlapping estimates were obtained every $0.125 \mathrm{~s}$, resulting in $1280 \mathrm{ZL}$ estimates. To separate the airway and tissue mechanics, the constant phase tissue model was fitted to each segmental ZL spectrum by minimizing the differences between the measured and modeled impedance values. A smoothing procedure applying a 50-point moving average was used for the time courses of the forced oscillatory model parameters. The airway parameters were corrected for the resistance and inertance of the endotracheal tube.

\subsubsection{Impedance measurements with the classical setup in patients}

To measure Zrs in patients during spontaneous breathing, a modified FOT system was used with a pseudorandom oscillatory pressure signal in the $4-26 \mathrm{~Hz}$ frequency range. The smallamplitude oscillations were led through a $22 \mathrm{~mm}$ inner diameter flexible tube and transmitted through a $28 \mathrm{~mm}$ inner diameter screen pneumotachograph connected to a differential pressure transducer (Validyne MP45; Validyne Engineering, Northridge, CA), which was used to sense input airflow (V'). The patient side of the pneumotachograph was attached to a disposable bacterial filter and a mouthpiece (Type PBF-30; Piston Ltd, Budapest, Hungary). A lateral port between the pneumotachograph and the bacterial filter was used to measure the airway opening pressure (Pao) with another pressure transducer (ICS model 33NA002D, ICSensors, Milpitas, CA).

Fourier transformation with $4 \mathrm{~s}$ time windows and 95\% overlapping was used to calculate Zrs $\left(\mathrm{Zrs}=\mathrm{Pao} / \mathrm{V}^{\prime}\right)$ from $12 \mathrm{~s}$ long recordings.

From the impedance measurements Raw was estimated by the average resistance values at the location where the real part of Zrs (Rrs) was reasonably frequency independent (16 to $26 \mathrm{~Hz}$ ). The elastance of the total respiratory system (E) was estimated by model fitting of the reactance (Xrs) from the imaginary part of Zrs through minimization of the differences between the measured and the modeled Xrs data:

$$
\mathrm{Xrs}=\mathrm{j}(\omega \mathrm{Iaw}-\mathrm{E} / \omega)
$$

Between the loudspeaker and the pneumotachograph a bias tube $(30 \mathrm{~cm}$ long and $1 \mathrm{~cm}$ in inner diameter) was attached to a side port which allowed the patients to breathe normally during the oscillatory measurements. The input impedance of the bacterial filter, the mouthpiece, and the connecting tubing was measured; Zrs recordings were corrected for this instrumental component. 


\subsection{Study protocols}

\subsubsection{Effects of pulmonary capillary pressure on lung mechanics}

In the isolated lung setup the perfusion of the lungs was established by applying physiological levels of Ppa and Pla which resulted in a Pc of $10 \mathrm{mmHg}$. The resulting flow $(6-8 \mathrm{ml} / \mathrm{min})$ was then kept constant for each lungs. During the experiments both Ppa and Pla were adjustable simultaneously to change the value of Pc, as performed previously [1]. Lungs were normoventilated at a PEEP of $2.5 \mathrm{cmH}_{2} \mathrm{O}$ and a tidal volume of $7 \mathrm{ml} / \mathrm{kg}$. The experimental protocol was started when the perfusion of the isolated lung was established and had become steady-state under this condition. In eight animals the Pc was then set to 0 (unperfused), 5, 10 or $15 \mathrm{mmHg}$ in random sequence by simultaneously changing the height of the venous and arterial containers to maintain a constant blood flow. Randomization of Pc was carried out to avoid any bias in the results due to temporal changes in lung mechanics during the perfusions. To standardize the volume history, hyperinflations were made prior to the measurements by occluding the expiratory port of the ventilator and then three ZL recordings were performed. The lungs were ventilated for $10 \mathrm{~min}$ at this PEEP level and at the end of this period another set of ZL was collected in order to assess the changes in airway and tissue mechanics during normoventilation. After completion of the ZL measurements, the pressure-volume curve of the lungs $(\mathrm{P}-\mathrm{V})$ was recorded, and this was followed by the recording of another set of ZL. These latter measurements allowed the assessment of the effects of a controlled recruitment maneuver on the lung mechanics in the presence of different Pc levels. The PEEP was then lowered to $0.5 \mathrm{cmH}_{2} \mathrm{O}$, and 1 min later the whole sequence of measurements was repeated. Following the measurements at the first Pc level, the PEEP was increased back to $2.5 \mathrm{cmH}_{2} \mathrm{O}$ and hyperinflations were administered to fully recruit the lungs and to standardize the volume history. The next level of Pc was then established and the same protocol at both PEEP levels was repeated until all four perfusion settings had been completed.

\subsubsection{Lung mechanical and vascular changes during positive- and negative- pressure lung inflations}

In the isolated lung setup after the steady-state condition had been reached, in eight animals, a slow (160-s) lung inflation-deflation maneuver was performed. First the maneuver was carried out with positive pressure (PPLI) through the wavetube by elevating the pressure in the loudspeaker chamber until a maximum transpulmonary pressure (Ptp) of $22 \mathrm{cmH}_{2} \mathrm{O}$ was attained, and passive expiration was then achieved by opening the loudspeaker chamber to the 
atmosphere via an adjustable leak. Following this maneuver, the box containing the heart-lung preparation was closed. A closed-circuit ventilator was then connected to the box, creating the same ventilation pattern (Ptp, VT, and ventilation frequency) as maintained during positivepressure ventilations by inducing negative pressures in the box. After the initiation of the negative-pressure ventilation a $10 \mathrm{~min}$ period was necessary to reach steady-state mechanical and hemodynamical conditions. Ppa and Pla were adjusted to maintain the same levels as during positive pressure ventilations. A slow (160-s) inflation-deflation maneuver (NPLI) was then achieved by gradually raising the vacuum in the box from -2.5 to $-22 \mathrm{cmH}_{2} \mathrm{O}$ while the trachea was open to the atmosphere (inflations), and the box pressure was allowed to reach the initial level by opening it via an adjustable leak (deflations). During negative-pressure maneuvers, the same inflation-deflation times were adjusted as during positive pressures. The impedance measurements during negative-pressure maneuvers incorporated the input impedance of the closed box therefore the box impedance alone was measured following the experiments and was subtracted as a serial impedance with the isolated lungs. Because the tracheal flow always passed through the wave tube, changes in lung volume during inflationdeflation maneuvers were assessed by integrating the pressure drop across the wave tube (P1 P2), as detailed previously [20]. Following completion of the experimental protocol, the inferior lobe of each lung was weighed, dried in an oven (Memmert, Schwabach, Germany) at $60{ }^{\circ} \mathrm{C}$ for 2 days, and weighed again to determine the wet-to-dry lung weight ratio.

\subsubsection{Bronchial hyper-responsiveness after coronary ischemia}

In the in vivo experiments when stable hemodynamic and respiratory mechanical conditions had been reached, four-to-six $6 \mathrm{~s}$ long Zrs measurements were recorded. Zrs was also measured after the iv. injection of serotonin at a dose of $20 \mu \mathrm{g} / \mathrm{kg}$ at $15 \mathrm{~s}$, followed by recordings at 1-min intervals until $10 \mathrm{~min}$. Fifteen minutes later, when the respiratory mechanics and systemic blood pressure had returned to the baseline, the iv. serotonin challenge was repeated at a dose of $30 \mu \mathrm{g} / \mathrm{kg}$, and the changes were followed with the same timing. The coronary artery was then prepared as described above, and myocardial ischemia was induced in the animals in Group I, while in the rats in Group C the silk thread was removed without the occlusion of the coronary artery. The thorax was then closed and the animals were awakened. Four weeks later, the rats were anaesthetized and intubated again in an identical manner as during the first experiments. The changes in the lung responsiveness to serotonin were assessed by repeating the constrictor challenges described earlier. At the end of the protocol, the chest was opened, and the end diastolic ventricular pressure (EDLVP) was 
measured by puncturing the left ventricle and connecting the needle to a water-filled pressure transducer. The lungs were then fixed by instilling $4 \%$ formalin into the trachea at a hydrostatic pressure of $20 \mathrm{cmH}_{2} \mathrm{O}$ and the lungs and the heart were dissected for histological investigations.

\subsubsection{Changes in airway and respiratory tissue mechanics after cardiac surgery}

Measurement Times and Protocol. In patients, four-to-six reproducible Zrs recordings were recorded prior to the premedication on the day before surgery. The patients wore a nose clip and were asked to breathe normally through the bias tube of the forced oscillatory system, with their cheeks supported by their palms. They were positioned in a $30^{\circ}$ supine position, similarly as in the postoperative measurements, so as to avoid potential bias due to different body postures. The Zrs data were subsequently collected in a subgroup of five patients in the sitting position in order to estimate the influence of the body position on the impedance parameters. After completion of the Zrs recordings, standard spirometry was performed in accordance with the joint European Respiratory Society-American Thoracic Society guidelines [43]. The first postoperative Zrs data were collected at the postoperative intensive care unit two to four hours after tracheal extubation (four to six hours after the surgery), when the patients were alert and their steady spontaneous breathing had returned. Their respiratory mechanical status was followed up by performing Zrs measurements twice a day (mornings and evenings) until their discharge from the hospital (day 6 postoperatively).

\subsection{Statistics}

Scatters in the parameters are expressed in standard error (SE) values. The KolmogorovSmirnov test was used to test data for normality. Paired t-tests were applied to assess statistical significance in different conditions in the same groups. One-way repeated measures analysis of variances (ANOVA) was applied to compare the relative changes in the measured parameters. Two-way (ANOVA) with variables was used to evaluate changes in different groups and parameters. Pairwise comparisons were performed by using the Student-NewmanKeuls multiple comparison procedures. Mann-Whitney rank sum test was performed for variables where the parameters were not distributed normally. Spearman correlation test was used to assess the strength of association between different variables. The influence of continuous variables on the changes of the measured parameters were examined by means of Pearson correlation tests. Statistical tests were carried out with a significance level at $p<0.05$. 


\section{Results}

\subsection{The influence of the pulmonary microvascular pressure on the mechanical properties of the lung}

Figure 2 depicts the airway and tissue parameters before and after the 10 min mechanical ventilation with the normal and a lowered PEEP, and following the recruitment maneuvers accomplished by slow inflations to $30 \mathrm{cmH}_{2} \mathrm{O}$.
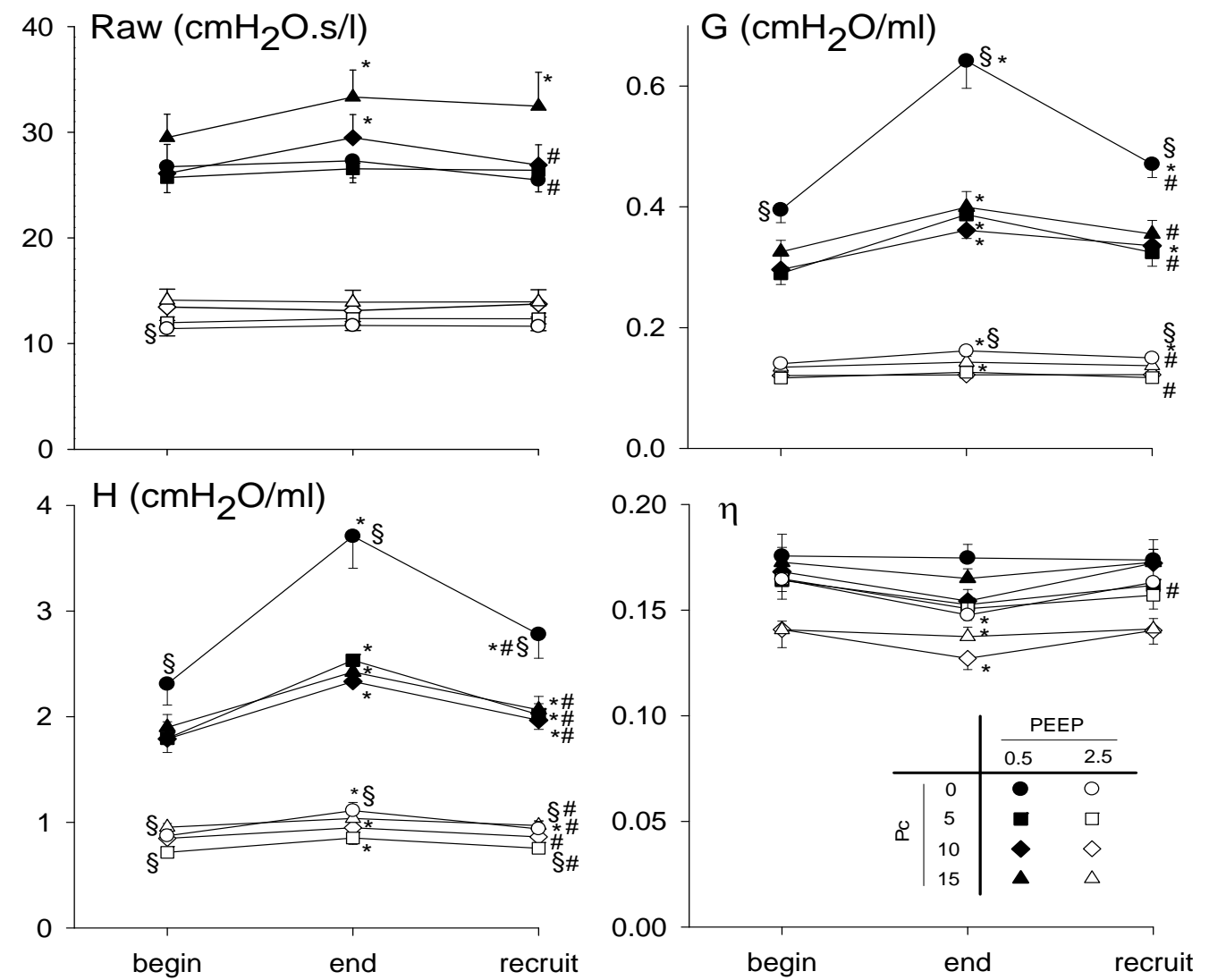

Figure 2. Airway resistance, lung tissue damping and elastance at the beginning (begin) and at the end (end) of a 10-min period and following a recruitment maneuver (recruit). PEEP 2.5 (open symbols) and $0.5 \mathrm{cmH}_{2} \mathrm{O}$ (closed symbols). (•) Pc $=0 \mathrm{mmHg},(\boldsymbol{\bullet}) \mathrm{Pc}=5 \mathrm{mmHg},(\diamond) \mathrm{Pc}=10 \mathrm{mmHg},(\boldsymbol{\Delta}) \mathrm{Pc}=15 \mathrm{mmHg} .{ }^{*} \mathrm{p}<0.05$ vs. begin; \#p $<0.05$ vs. end; $\S p<0.05$ vs. the corresponding parameter value at a Pc of $10 \mathrm{mmHg}$ within a PEEP.

At the normal PEEP, no significant change in Raw occurred during the experiments, while ventilation using a low PEEP led to slight, but statistically significant increases in Raw while Pc levels of 10 and $15 \mathrm{mmHg}$ were maintained. The increase was sustained after the recruitment maneuvers at the Pc of $15 \mathrm{mmHg}$, whereas it was fully reversible at the Pc of 10 mmHg. The maintenance of zero or low lung perfusion pressures was associated with significant elevations in the lung tissue parameters after the mechanical ventilation; these changes were greatly exaggerated when the PEEP was lowered, and in particular at $\mathrm{Pc}=0 . \eta$ was fairly constant throughout the experiments. 
The relative changes in the airway and lung tissue parameters following the mechanical ventilation and after the recruitment maneuvers are shown in Figure 3, different perfusion pressures being maintained in the pulmonary capillaries.
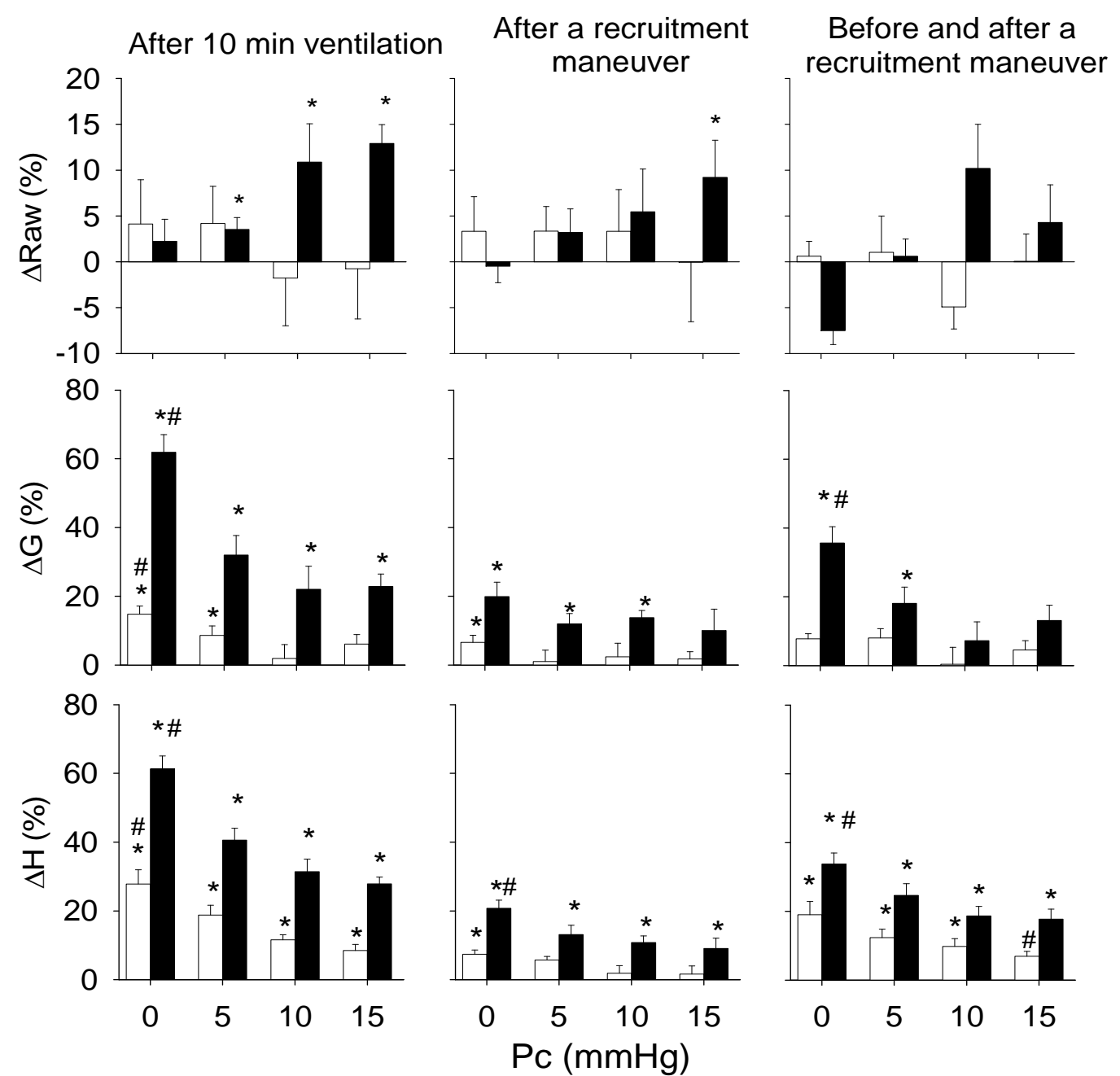

Figure 3. Relative changes in the mechanical parameters as a function the capillary pressure $(\mathrm{Pc})$ following mechanical ventilation (left panels), after recruitment maneuvers relative to the initial condition (middle panels), and before and after the recruitment maneuvers (right panels). PEEP 2.5 (hollow bars); $0.5 \mathrm{cmH}_{2} \mathrm{O}$ (filled bars). *Significant change $(\mathrm{p}<0.05)$; $\# \mathrm{p}<0.05$ vs. the parameter value at $\mathrm{Pc}=10 \mathrm{mmHg}$.

At a PEEP of $2.5 \mathrm{cmH}_{2} \mathrm{O}$, significant change in Raw was not observed at any Pc level, whereas lowering the PEEP led to significant increases in Raw in the perfused lungs. The parenchymal parameters display similar, but greater changes at low than at higher PEEP, i.e. a tendency to more pronounced increases in $\mathrm{G}$ and $\mathrm{H}$ with decreasing Pc (left panels). Recruitment maneuvers lowered the elevated levels of $\mathrm{G}$ and $\mathrm{H}$, but with no obvious Pc dependence when these changes are related to the initial conditions (middle panels). The changes in the tissue mechanical parameters before and after the $\mathrm{P}-\mathrm{V}$ curve recordings were greater at low prefusion pressures (right panels). 
$\mathrm{P}-\mathrm{V}$ curves obtained following the mechanical ventilation period at different Pc levels are exemplified in Figure 4 for a representative isolated perfused lung.
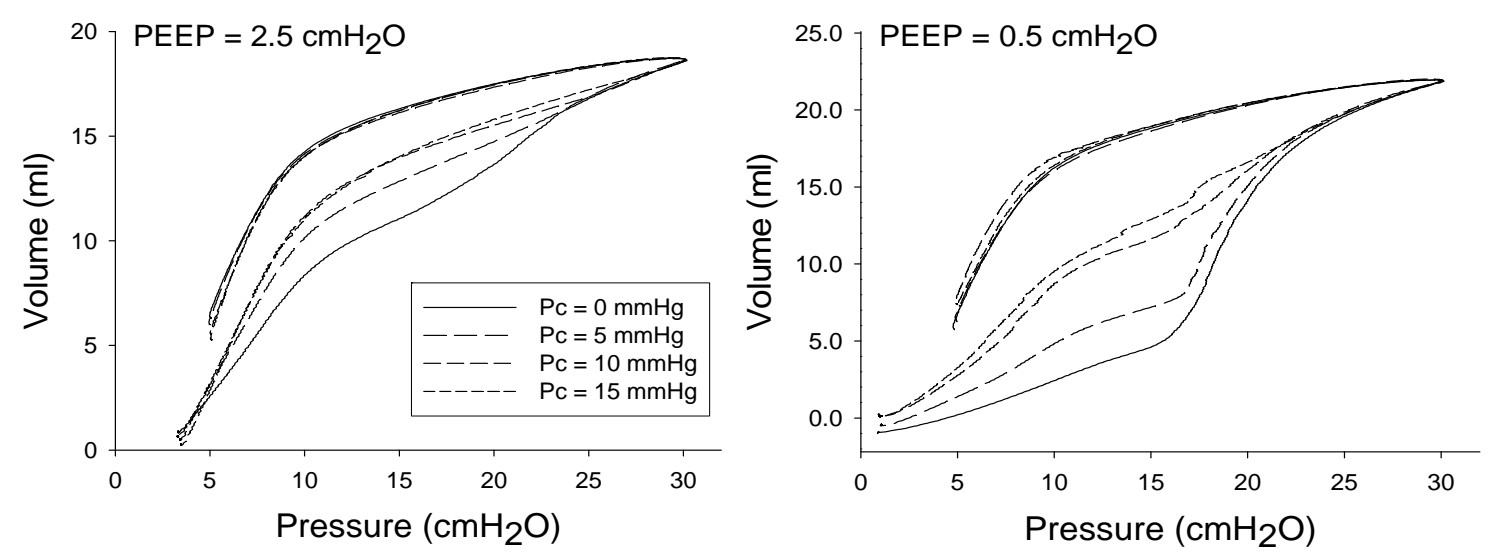

Figure 4. Pressure-volume curves obtained in a representative isolated perfused lung following the mechanical ventilation period from a PEEP of 2.5 (left) or $0.5 \mathrm{cmH}_{2} \mathrm{O}$ (right) while different levels of $\mathrm{Pc}$ are maintained.

Lowering of Pc to below its physiological level induced marked changes in the shape of the $\mathrm{P}-\mathrm{V}$ curve, with the appearance of the lower inflection point during inflations, particularly at a PEEP of $0.5 \mathrm{cmH}_{2} \mathrm{O}$.
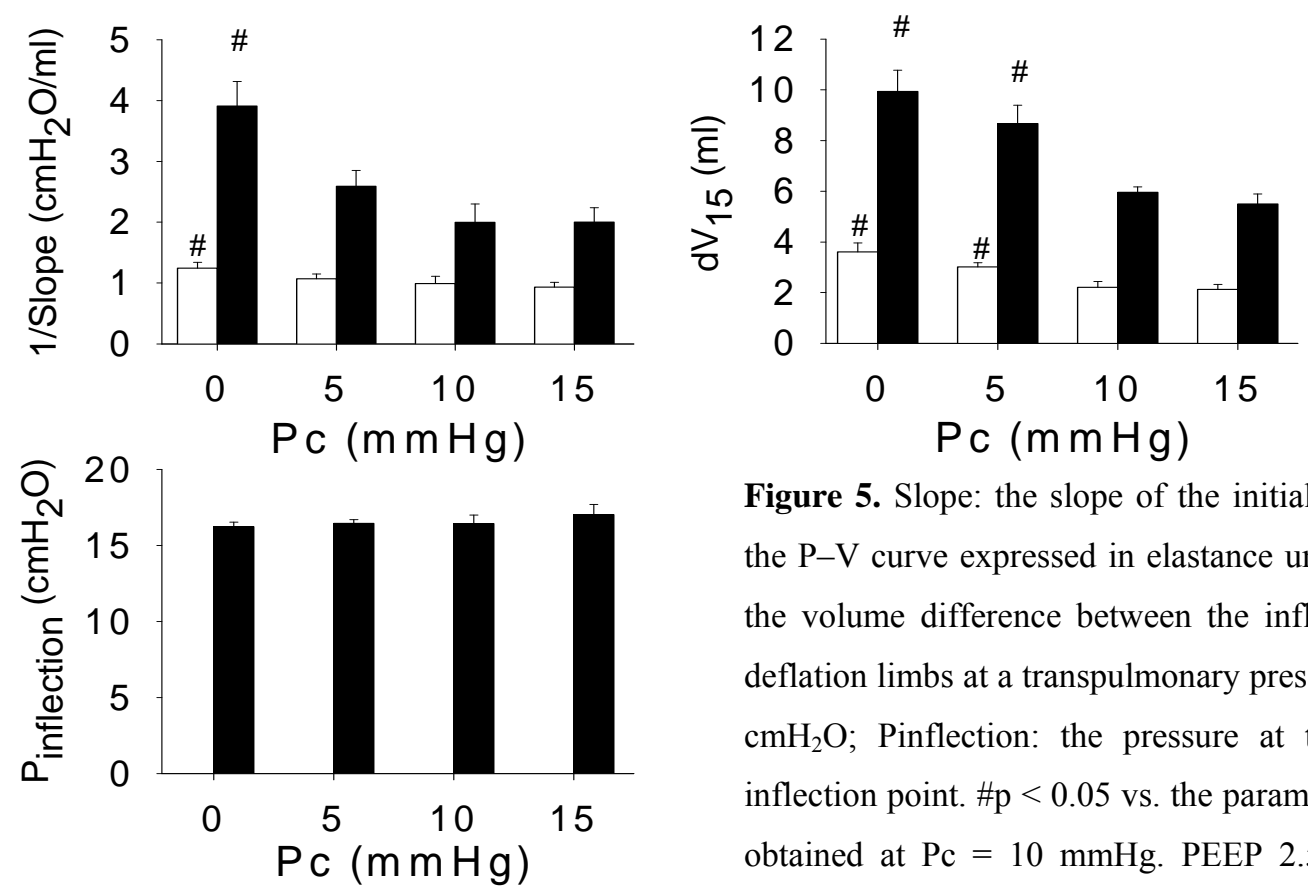

Figure 5. Slope: the slope of the initial phase of the $\mathrm{P}-\mathrm{V}$ curve expressed in elastance unit; dV15: the volume difference between the inflation and deflation limbs at a transpulmonary pressure of 15 $\mathrm{cmH}_{2} \mathrm{O}$; Pinflection: the pressure at the lower inflection point. $\# \mathrm{p}<0.05$ vs. the parameter value obtained at Pc $=10 \mathrm{mmHg}$. PEEP 2.5 (hollow bars); $0.5 \mathrm{cmH}_{2} \mathrm{O}$ (filled bars)

Figure 5 illustrates the relationships between the indices extracted from the $\mathrm{P}-\mathrm{V}$ curves and the pulmonary perfusion pressure. Similarly to the parenchymal mechanical parameters, the level of Pc maintained during perfusion markedly affected the slope of the inflation limb of the $\mathrm{P}-\mathrm{V}$ curve and the volume difference between the inflation and deflation limbs (dV15), influence being more pronounced at a low PEEP level. The changes in Pc had no systematic effects on the pressure read at the lower inflection point. 


\subsection{The effect of positive- and negative-pressure lung inflations on lung mechanics and the pulmonary vasculature}

Temporal changes in Rv and in the mechanical parameters obtained during PPLI and NPLI maneuvers in a representative lung are demonstrated in Figure 6. Similar changes were observed in the mechanical parameters, while Rv exhibited increases during PPLI and decreased slightly with NPLI.
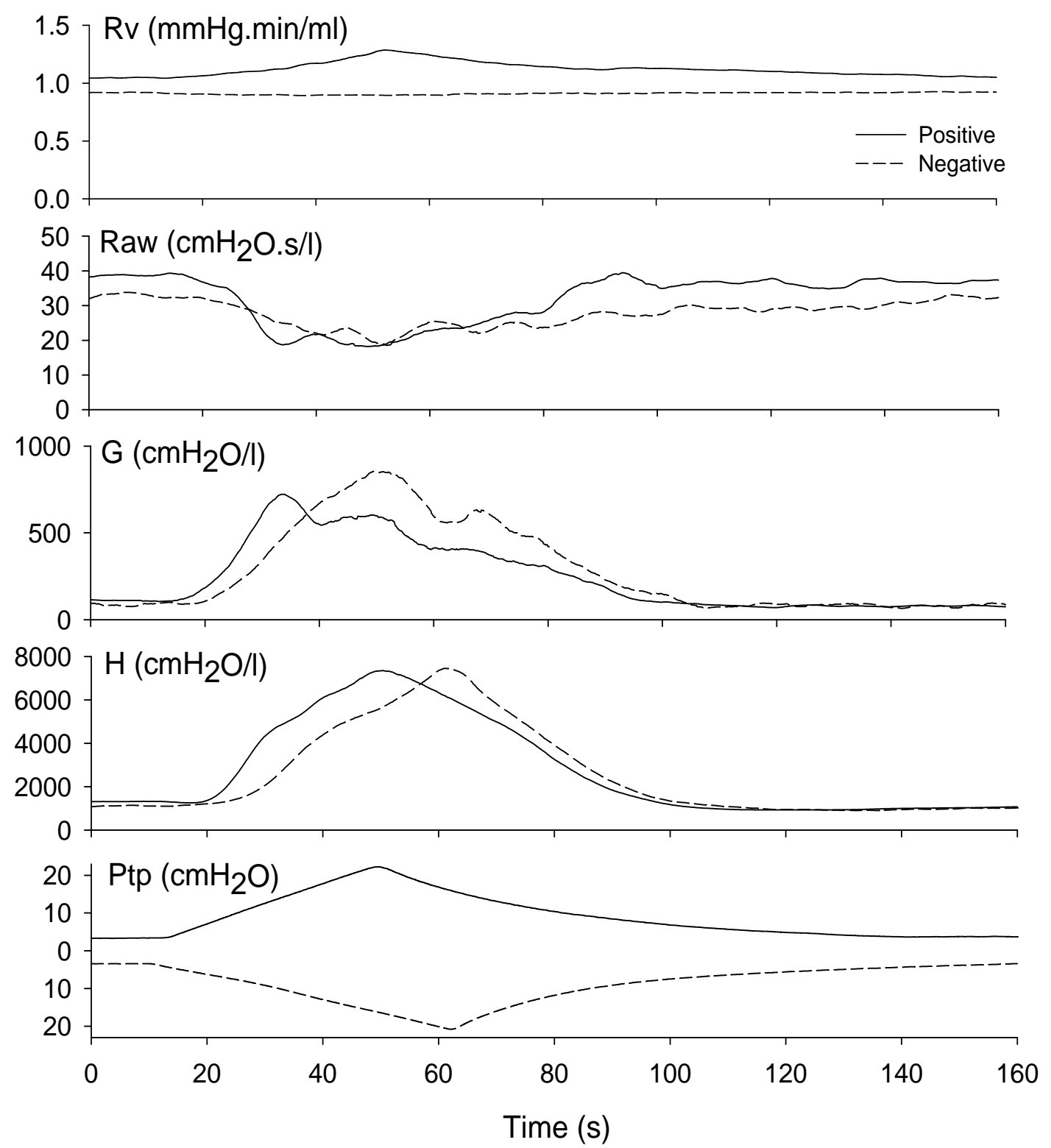

Figure 6. Changes in pulmonary vascular resistance (Rv) and in the mechanical parameters during lung inflation-deflation maneuvers with positive airway pressure and with the generation of negative pressure around the pleura in a representative lung. Raw, airway resistance; G, tissue damping; H, tissue elastance; Ptp, transpulmonary pressure. 
Changes in the airway and parenchymal mechanical parameters during PPLI and NPLI maneuvers are depicted in Figure 7. The two different means of lung inflations had no influence on the course of the mechanical parameters: the decreases in Raw were associated with marked increases in G and H. Furthermore, the lung tissue parameters were significantly lower during deflations than at the same pressures during inflations $(\mathrm{p}=0.008$ and $\mathrm{p}<0.001$ for $\mathrm{G}$, and $\mathrm{p}<0.05$ and $\mathrm{p}<0.001$ for $\mathrm{H}$ at a Ptp of $10 \mathrm{cmH}_{2} \mathrm{O}$ ).

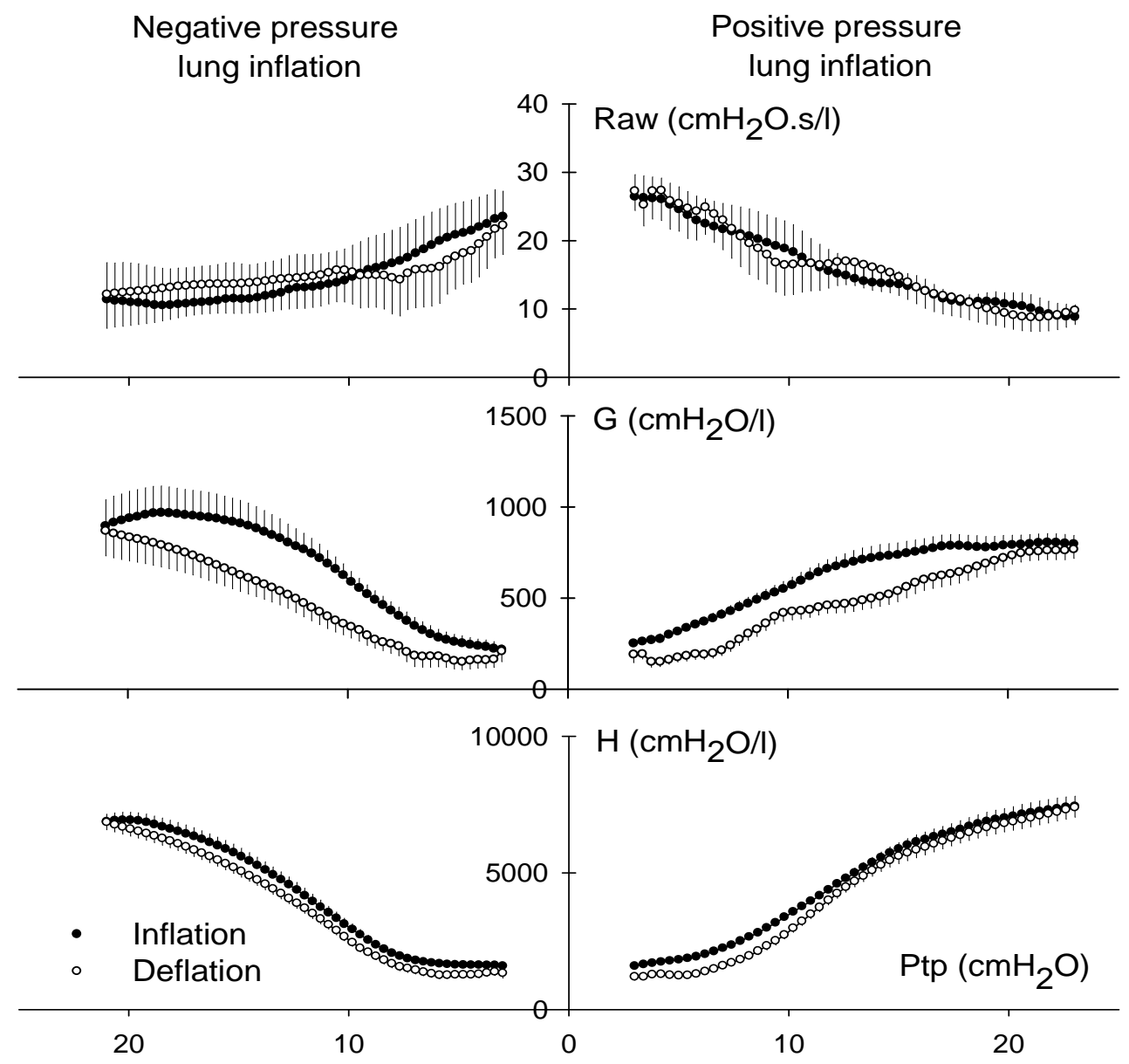

Figure 7. Changes in the airway and parenchymal mechanical parameters as functions of Ptp when the lungs are inflated with positive (right) or negative pressures (left). F, Inflations; E, Deflations.

In contrast with the mechanical parameters, $\mathrm{Rv}$ exhibited opposite changes in response to PPLI and NPLI (Figure 8), with marked increases during PPLI and minor decreases when NPLI maneuvers were performed. During PPLI, the Rv-Ptp curve exhibited hysteresis opposite to that observed for the tissue mechanical parameters; i.e., Rv was statistically significantly greater during deflation than during inflation $\left(\mathrm{p}<0.001\right.$ at a Ptp of $\left.10 \mathrm{cmH}_{2} \mathrm{O}\right)$, whereas such hysteresis was not observed for NPLI $\left(\mathrm{p}=0.16\right.$ at a Ptp of $\left.10 \mathrm{cmH}_{2} \mathrm{O}\right)$. Differences in $\mathrm{Rv}$ between the inflation and deflation limbs were not apparent when the changes in $\mathrm{Rv}$ were expressed as a function of lung volume. 


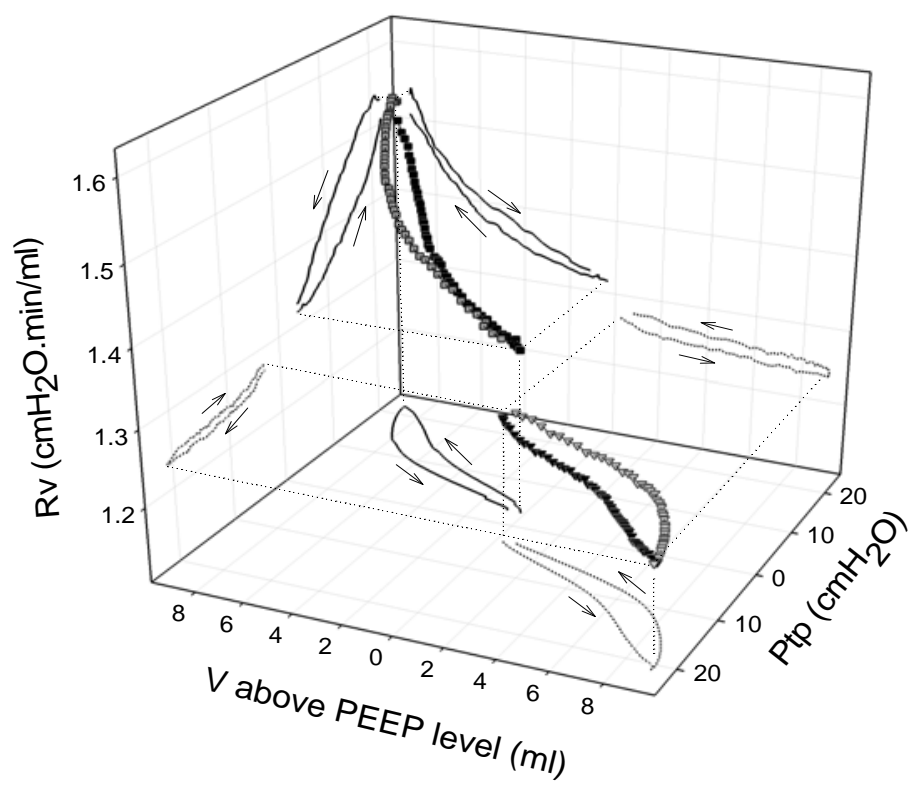

Figure 8. Changes in $\mathrm{Rv}$ as a function of the Ptp (left plane) and as a function of the lung volume ( $\mathrm{V}$; back plane) when the lungs are inflated with positive (squares) or negative pressures (triangles). Solid symbols: inflations; gray symbols: deflations; solid lines: projection curves of the positivepressure lung inflation maneuvers; dotted lines: projection curves of the negative-pressure lung inflation maneuvers.

Figure 9 depicts the mechanical parameters and Rv obtained in the inflation limb of the PPLI and NPLI maneuvers at particular Ptp levels.
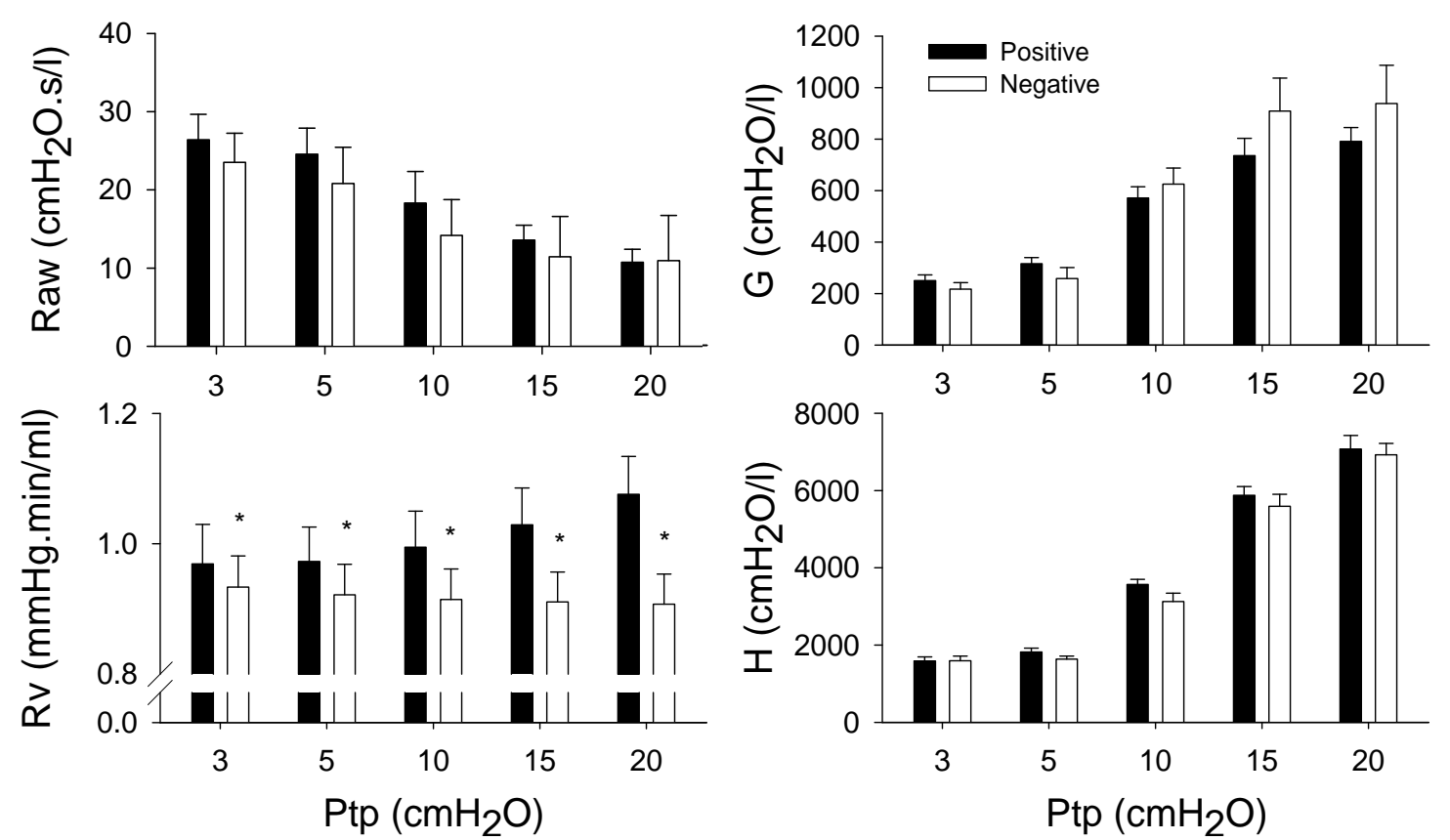

Figure 9. Lung mechanical parameters (Raw, $\mathrm{G}$, and $\mathrm{H}$ ) and Rv at different Ptp during positive- (filled bars) and negative-pressure (open bars) lung inflations. ${ }^{*} \mathrm{p}<0.05$ positive vs. negative.

Two-way ANOVA revealed no significant interactions between the factors, i.e., Ptp and the mode of inflation ( $\mathrm{p}=0.613, \mathrm{p}=0.37$, and $\mathrm{p}=0.141$ for Raw, $\mathrm{G}$, and $\mathrm{H}$, respectively), suggesting the lack of differences in the pressure dependent changes in the mechanical parameters between the NPLI and PPLI maneuvers. In contrast, these factors exhibited a highly significant interaction $(\mathrm{p}<0.001)$, demonstrating that the mode of inflation had a significant impact on the changes in Rv. 


\subsection{Airway hyper-responsiveness following coronary ischemia}

There was no significant difference in body weight between the two protocol groups. Coronary ischemia led to necrosis in the myocardium (affecting $8.8 \pm 2.6 \%$ of the total myocardial area), and sustained elevations in EDLVP $(8.4 \pm 0.2 \mathrm{mmHg}$ vs. $18.4 \pm 1.7 \mathrm{mmHg}$ in groups $\mathrm{C}$ and $\mathrm{I}$, respectively, $\mathrm{p}<0.001$ ), indicating the occurrence of left ventricular failure.
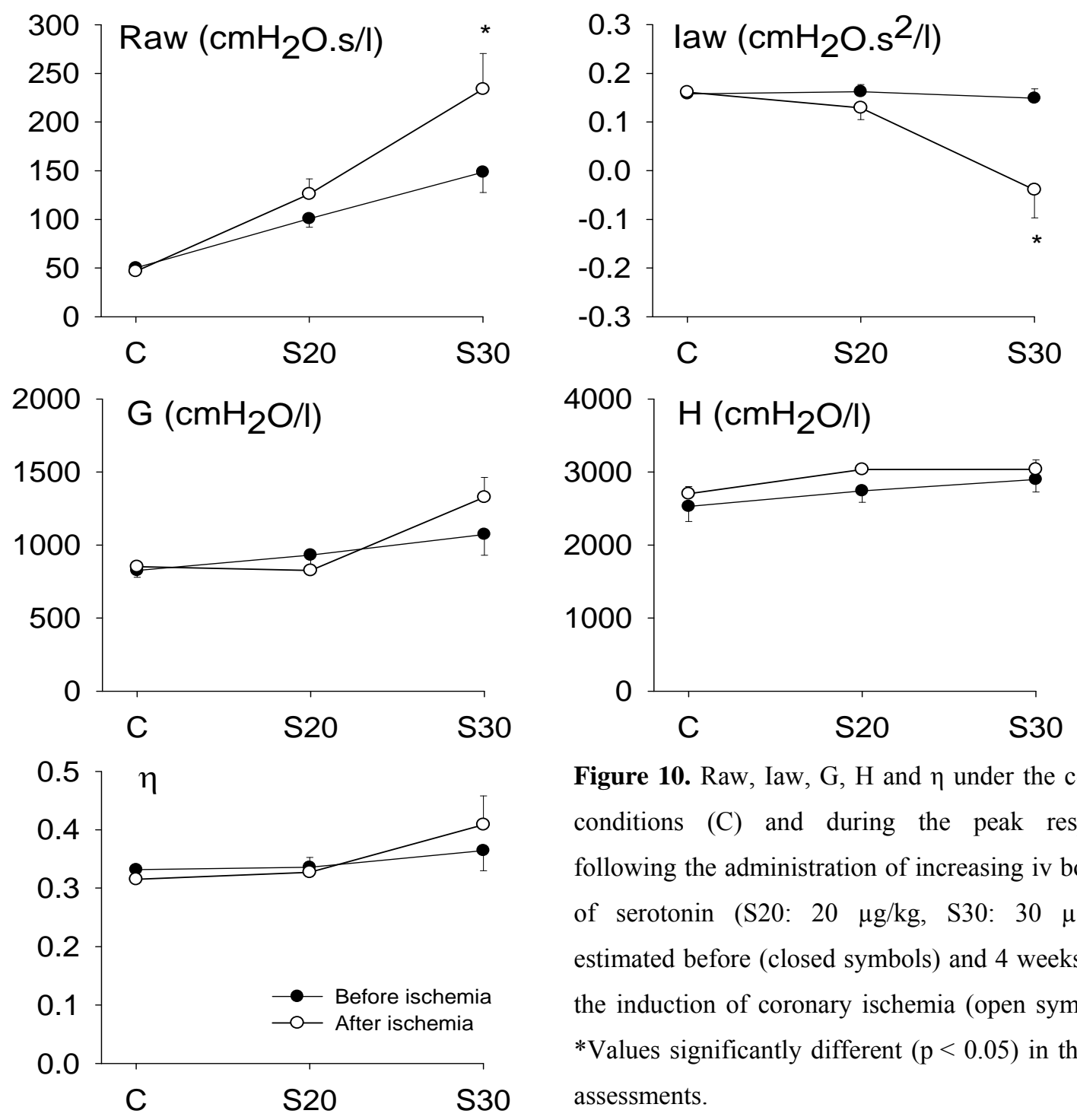

Figure 10. Raw, Iaw, G, $H$ and $\eta$ under the control conditions (C) and during the peak response following the administration of increasing iv boluses of serotonin (S20: $20 \mu \mathrm{g} / \mathrm{kg}, \mathrm{S} 30: 30 \mu \mathrm{g} / \mathrm{kg})$, estimated before (closed symbols) and 4 weeks after the induction of coronary ischemia (open symbols). *Values significantly different $(\mathrm{p}<0.05)$ in the two assessments.

The airway and respiratory tissue mechanical parameters under the control conditions and following the serotonin challenges before and after coronary ischemia in the animals in Group I are displayed in Figure 10. The presence of coronary ischemia during the repeated measurements ( 4 weeks after the first phase of the study) did not have a significant effect on the baseline values of Raw $\left(48.9 \pm 4.0 \mathrm{cmH}_{2} \mathrm{Os} / 1\right.$ vs. $46.6 \pm 2.1 \mathrm{cmH}_{2} \mathrm{O} . \mathrm{s} / 1$ for groups $\mathrm{C}$ and I respectively; $\mathrm{p}=0.6), \mathrm{G}\left(878 \pm 48 \mathrm{cmH}_{2} \mathrm{O} / 1\right.$ vs. $\left.852 \pm 36 \mathrm{cmH}_{2} \mathrm{O} / \mathrm{l} ; \mathrm{p}=0.6\right)$ or $\mathrm{H}$ $\left(2694 \pm 113 \mathrm{cmH}_{2} \mathrm{O} / 1\right.$ vs. $\left.2703 \pm 98 \mathrm{cmH}_{2} \mathrm{O} / 1 ; \mathrm{p}=0.9\right)$. Serotonin induced an elevation in Raw, 
while the tissue mechanical parameters did not exhibit statistically significant changes after the challenges. The serotonin-induced elevations in Raw and the decreases in Iaw were more pronounced after left-heart failure induction $(\mathrm{p}=0.026$ and $\mathrm{p}=0.016$ for Raw and Iaw, respectively) following the $30 \mu \mathrm{g} / \mathrm{kg}$ dose).

The relative changes in the airway and tissue parameters at peak response following 20 and $30 \mu \mathrm{g} / \mathrm{kg}$ serotonin challenges are presented in Figure 11.
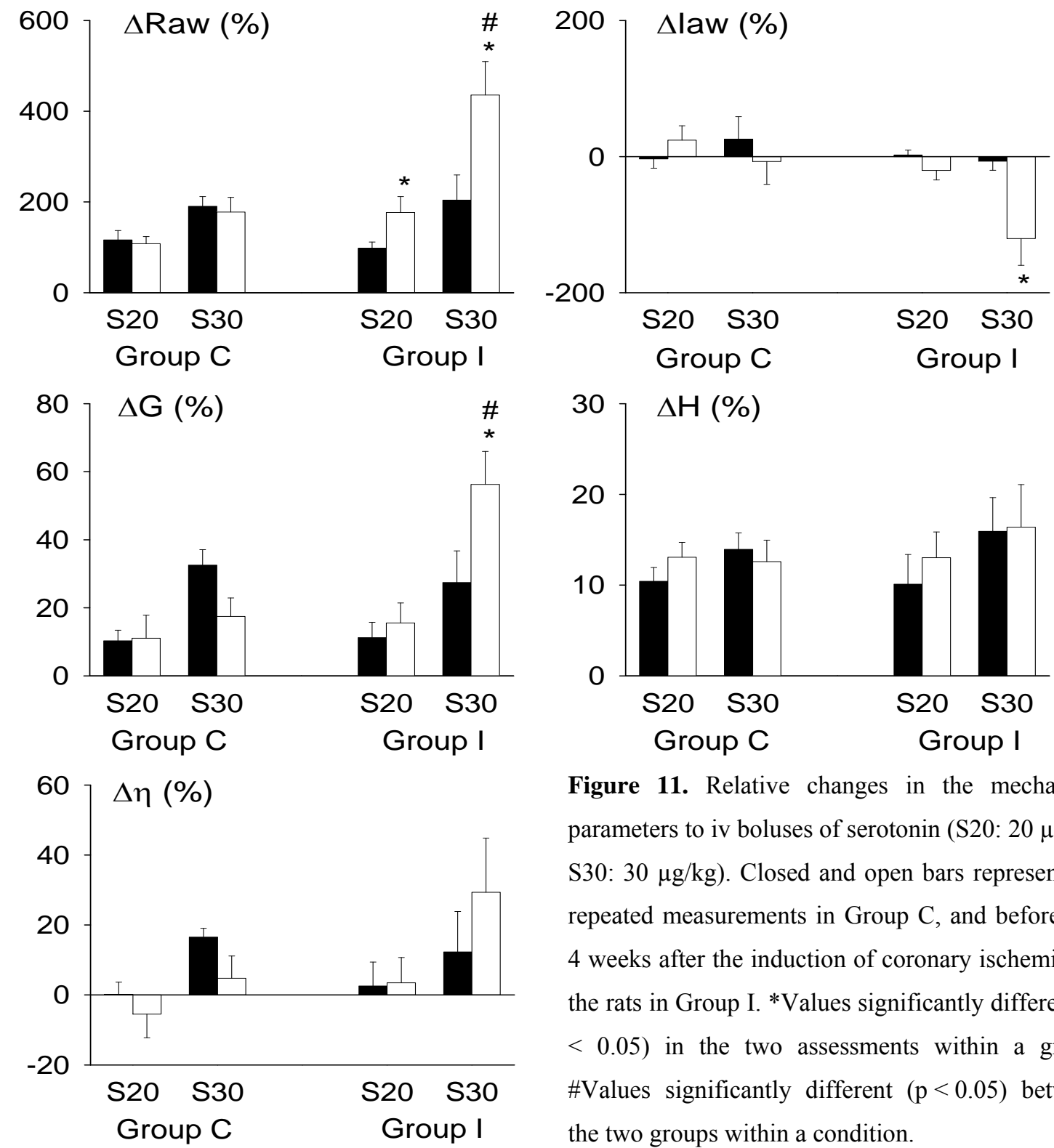

Figure 11. Relative changes in the mechanical parameters to iv boluses of serotonin (S20: $20 \mu \mathrm{g} / \mathrm{kg}$, S30: $30 \mu \mathrm{g} / \mathrm{kg}$ ). Closed and open bars represent the repeated measurements in Group $\mathrm{C}$, and before and 4 weeks after the induction of coronary ischemia for the rats in Group I. *Values significantly different (p $<0.05)$ in the two assessments within a group; \#Values significantly different $(p<0.05)$ between the two groups within a condition.

Reproducible elevations were observed in the respiratory mechanical parameters (Raw, G and $\mathrm{H})$ during the two serotonin provocations in the animals in Group C. In contrast, coronary ischemia led to significantly greater responses in Raw, Iaw and G, the predominant changes being observed in the airway mechanics. Serotonin had only minor effects on $\mathrm{H}$ and $\eta$; the changes in these parameters were not affected by the presence of lung congestion. In addition, 
further experiments were performed on nine more rats to investigate the possible involvement of the altered serotonin induced systemic blood pressure changes following coronary ischemia. These experiments revealed that the presence of coronary ischemia had no effect on the serotonin-induced decreases in the systemic blood pressure $(-19.4 \pm 3.2 \%$ vs. $-19.9 \pm 3.3 \%$ at $15 \mathrm{~s}$ after $20 \mu \mathrm{g} / \mathrm{kg}(\mathrm{p}=0.52)$, and $-16.2 \pm 2.3 \% \mathrm{vs} .-17.8 \pm 2.3 \%(\mathrm{p}=0.68)$ at $15 \mathrm{~s}$ after $30 \mu \mathrm{g} / \mathrm{kg}$ before and after coronary ischemia, respectively).

The relationship between EDLVP as an indicator of Pla and the enhanced responsiveness of Raw to serotonin is displayed in Figure 12 for the animals in group I, where the measurement of EDLVP was successful. No elevations in the lung responsiveness to serotonin was observed with increasing $\operatorname{EDLVP}\left(\mathrm{R}^{2}=0.087, \mathrm{p}=0.38\right.$ for all data, $\mathrm{R}^{2}=0.81$, $\mathrm{p}=0.08$ for the $20 \mu \mathrm{g} / \mathrm{kg}$ serotonin, and $\mathrm{R}^{2}=0.09, \mathrm{p}=0.68$ for the $30 \mu \mathrm{g} / \mathrm{kg}$ serotonin data).

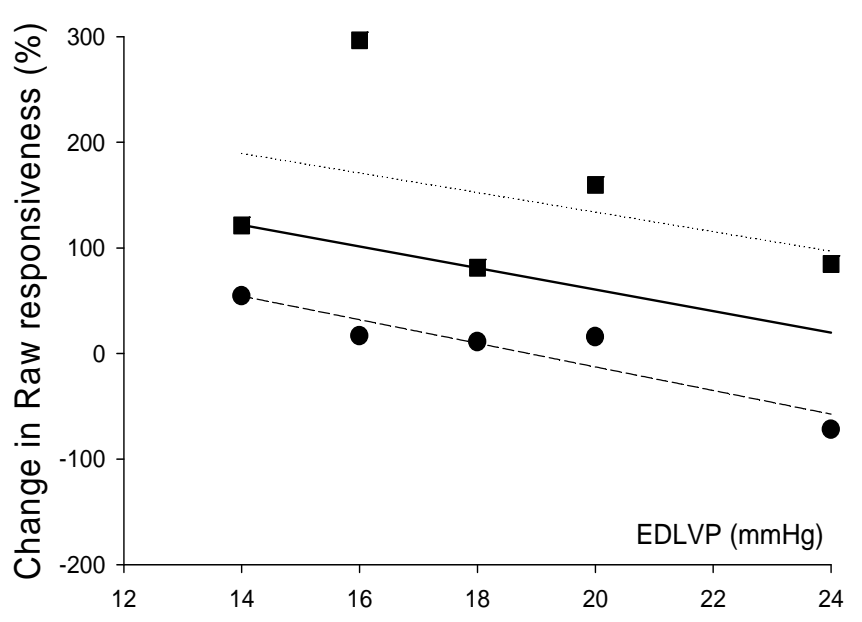

Figure 12. Relationship between end-diastolic left-ventricular pressure (EDLVP) and the alterations in the responsiveness in Raw to 20 (circles) and $30 \mu \mathrm{g} / \mathrm{kg}$ (squares) serotonin for the animals in Group I and 4 weeks after the first surgery. Solid line: best linear fit for all data; dashed line: best linear fit for $20 \mu \mathrm{g} / \mathrm{kg}$ serotonin data; dotted line: best linear fit for $30 \mu \mathrm{g} / \mathrm{kg}$ serotonin data.

Representative histological sections are demonstrated in Figure 13 in lungs obtained from rats in Groups C and I. Coronary ischemia led to an increased number of pericytes stained with the alpha smooth muscle actin ( $\alpha$-SMA) antibody in the alveolar walls.
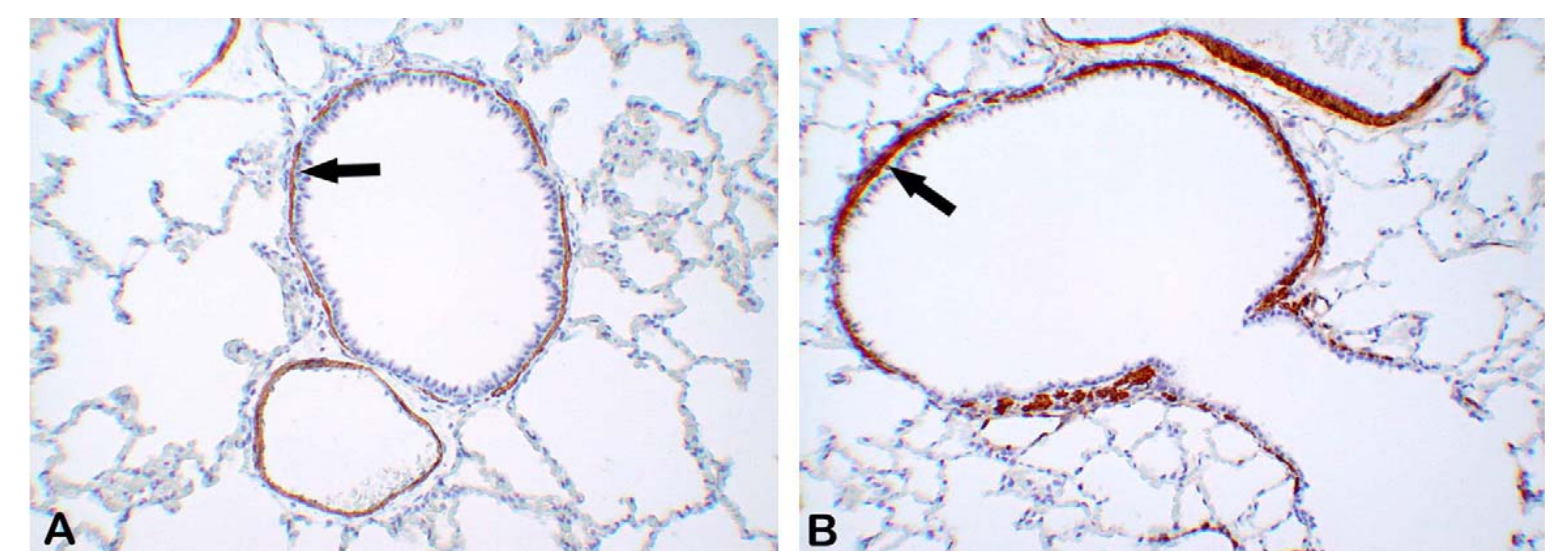

Figure 13. Lung histological sections obtained from a representative control rat (A) and rat with myocardial ischemia (B). In the terminal bronchial walls, the smooth muscle bundles (arrows) are much thicker in ischemic (B) than in the control (A) rats (slides were immunostained with an antibody against alpha smooth muscle actin, original magnification $200 \times$ ) . 
Figure 14 depicts the histological findings in the rats in Groups $\mathrm{C}$ and I. Left-heart failure induced a significant thickening of the pulmonary arteries and resulted in the development of perivenous and periarterial edema.
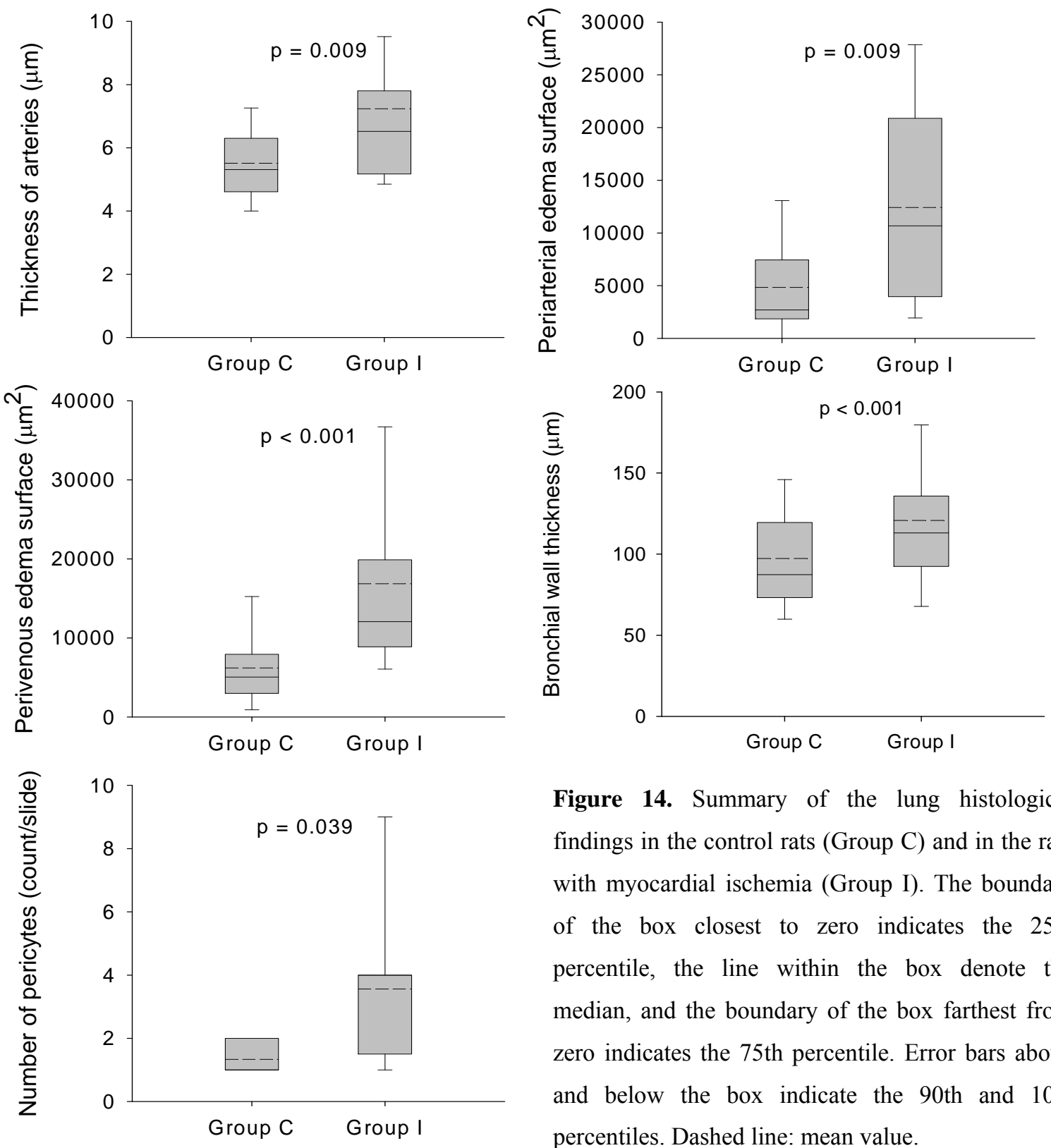

Figure 14. Summary of the lung histological findings in the control rats (Group C) and in the rats with myocardial ischemia (Group I). The boundary of the box closest to zero indicates the 25 th percentile, the line within the box denote the median, and the boundary of the box farthest from zero indicates the 75 th percentile. Error bars above and below the box indicate the 90th and 10th percentiles. Dashed line: mean value.

The bronchial wall thickness was significantly increased by the presence of coronary ischemia. Moreover, chronic lung congestion led to a proliferation of cells stained with $\alpha$ SMA antibody in the lungs, with significant increases in the number of positive $\alpha$-SMA cells in the alveolar walls and in the surface area of the peribronchial $\alpha$-SMA cells. 


\subsection{The effect of cardiac surgery on the mechanics of the respiratory system}

Representative Zrs Data. The Zrs curves obtained in a representative OPCAB patient before and after the cardiac surgery are demonstrated in Figure 15.

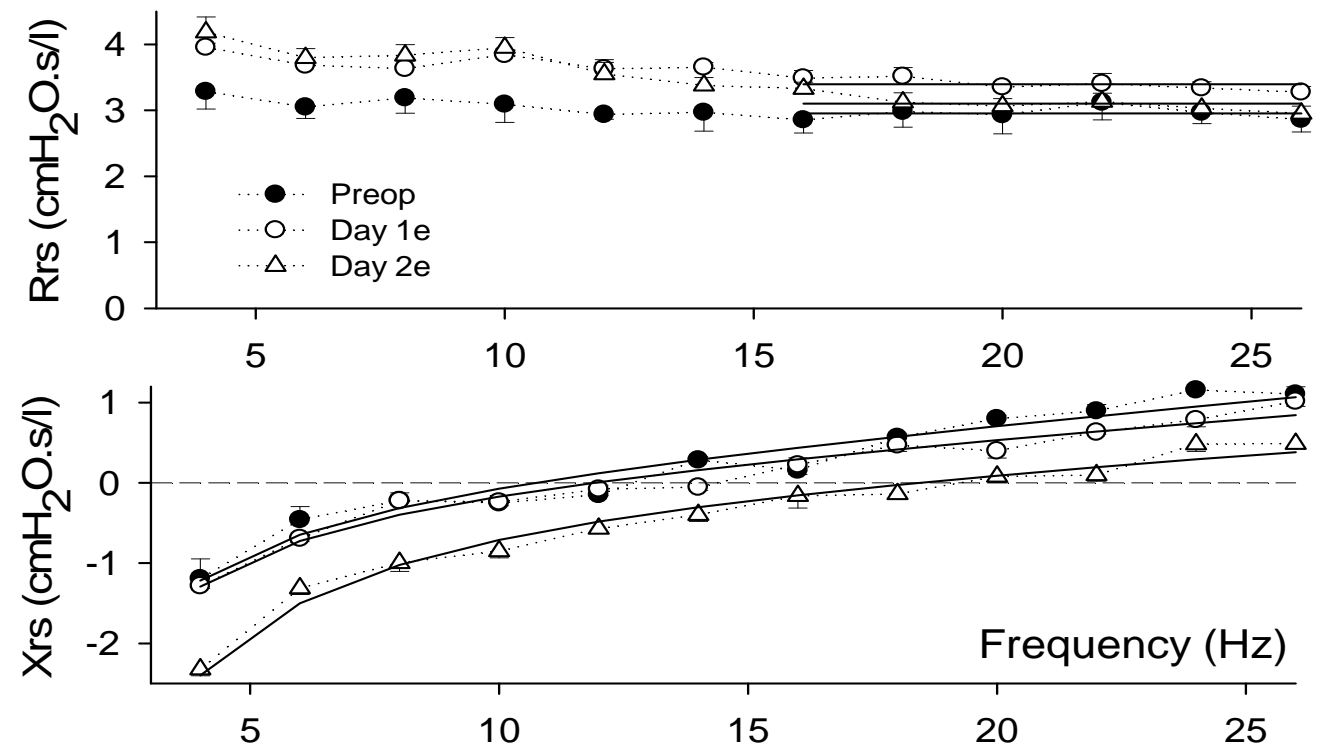

Figure 15. Real (Rrs) and imaginary (Xrs) parts of the input impedance (Zrs) in a representative patient before surgery $(\bullet)$, and during the evening of postoperative day $1(\circ)$ and $2(\Delta)$. Solid lines on Rrs denote the average of resistance between 16 and $26 \mathrm{~Hz}$ corresponding to Raw; solid lines on Xrs demonstrate the model fits.

The Rrs exhibited mild decreases in the lower frequency range but was fairly frequency independent at higher rates. The Xrs was predominantly characterized by hyperbolic increases reflecting the contribution of the elasticity of the respiratory tissues in the lower frequency range, while the approximately linear increases at higher frequencies revealed the contribution of inertance. The close to parallel increases in Rrs during the evening of the first postoperative day reflected airway narrowing with minor changes in Xrs, suggesting no alterations in the respiratory elastance. On the evening of the second postoperative day the decreases in Xrs suggested an enhancement in the elastic properties of the respiratory tissues. The model fitted the Xrs data well with an average fitting error of $0.15 \pm 0.01 \mathrm{cmH}_{2} \mathrm{O} . \mathrm{s} / \mathrm{l}$.

Time Course of the Changes in Respiratory Mechanical Parameters The changes in the airway and respiratory tissue mechanical parameters for the overall entire study group are demonstrated in Figure 16. An airway narrowing was observed immediately postoperatively, which was followed by a gradual return toward the preoperative level. Gradual increases in the elastance parameters were observed after the cardiac surgery with peak changes occurring around the two to three days postoperatively. Whereas Raw and Iaw fully returned to the normal level, E remained elevated systematically at the end of the study period. 


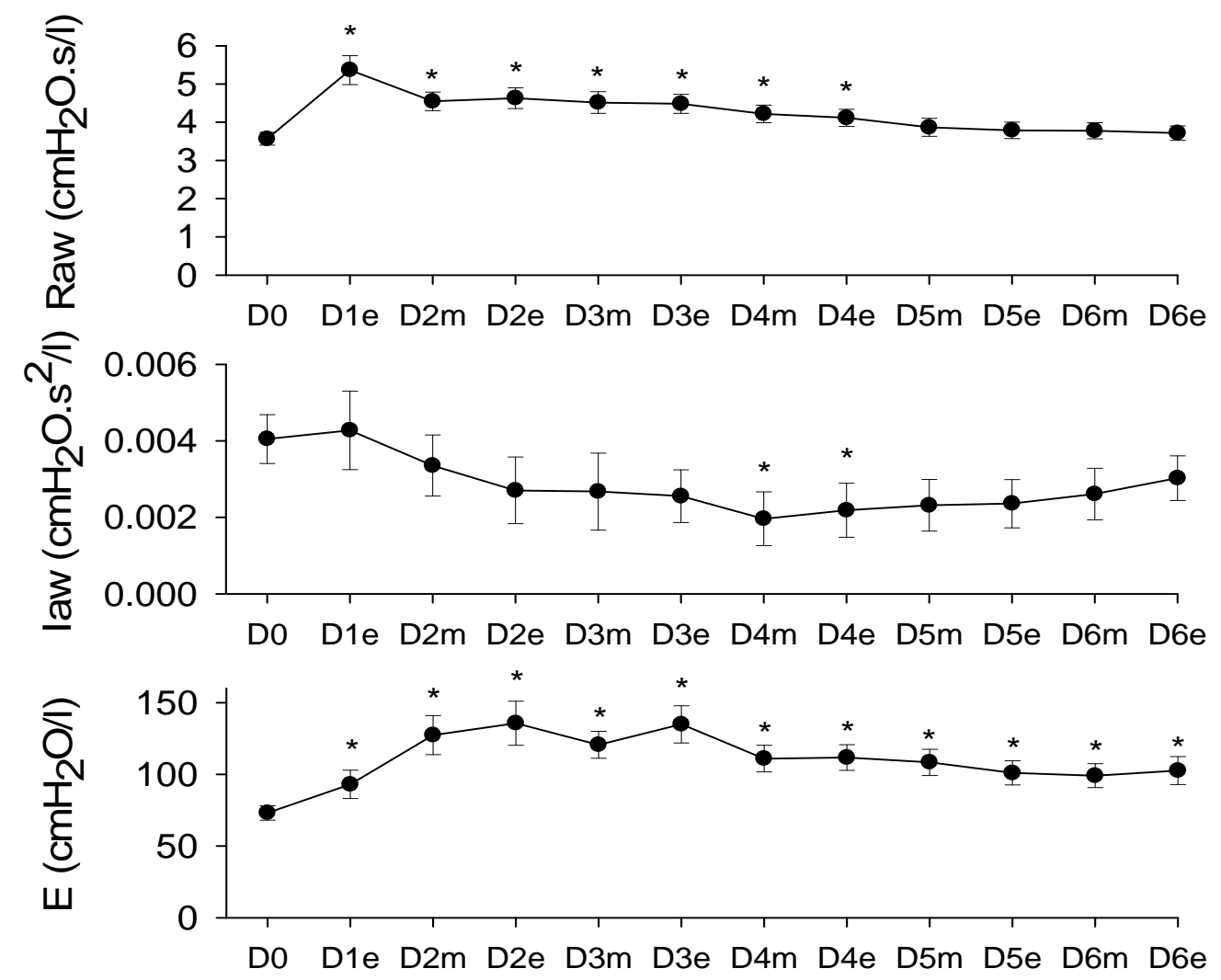

Figure 16. Airway resistance (Raw), inertance (Iaw), and respiratory elastance (E) before (day $0=\mathrm{D} 0)$ and after cardiac surgery for the overall study population (D1e to D6e; $\mathrm{m}=$ morning; $\mathrm{e}=$ evening; $*$ : $<0.05$ versus the parameter values observed on D0.)

Factors Affecting the Postoperative Changes in the Respiratory Mechanics. There were no significant interactions between the postoperative changes in the respiratory mechanical parameters and the gender $(\mathrm{p}=0.17, \mathrm{p}=0.18$, and $\mathrm{p}=0.49$ for Raw, Iaw and $\mathrm{E}$, respectively) or smoking habit ( $p=0.37, p=0.94$, and $p=0.91$, for Raw, Iaw, and $E$ ) or the duration of surgery $(R=-0.075, p=0.66, R=0.04, p=0.4$, and $R=0.072, p=0.68$ for Raw, Iaw, and $E$, respectively) or the age of the patients $(R=-0.21, p=0.21, R=0.18, p=0.31$, and $\mathrm{R}=-0.15, \mathrm{p}=0.38$ for Raw, Iaw, and $\mathrm{E}$ ), indicating that these factors do not contribute to the deterioration of the respiratory mechanics during the first week after cardiac surgery. In contrast, the detection of significant interactions clearly demonstrated that CPB strongly affected the postoperative changes in Raw $(p<0.001)$ and $E(p<0.05)$. Obesity similarly had a statistically significant impact on the elevation in E after the cardiac surgery $(p<0.005)$, though it merely tended to influence the increase in Raw $(\mathrm{p}=0.081)$.

Effects of CBP. The airway and respiratory tissue parameters before the operation and during the six-day follow-up after the cardiac surgery with or without CPB are illustrated in Figure 17. 


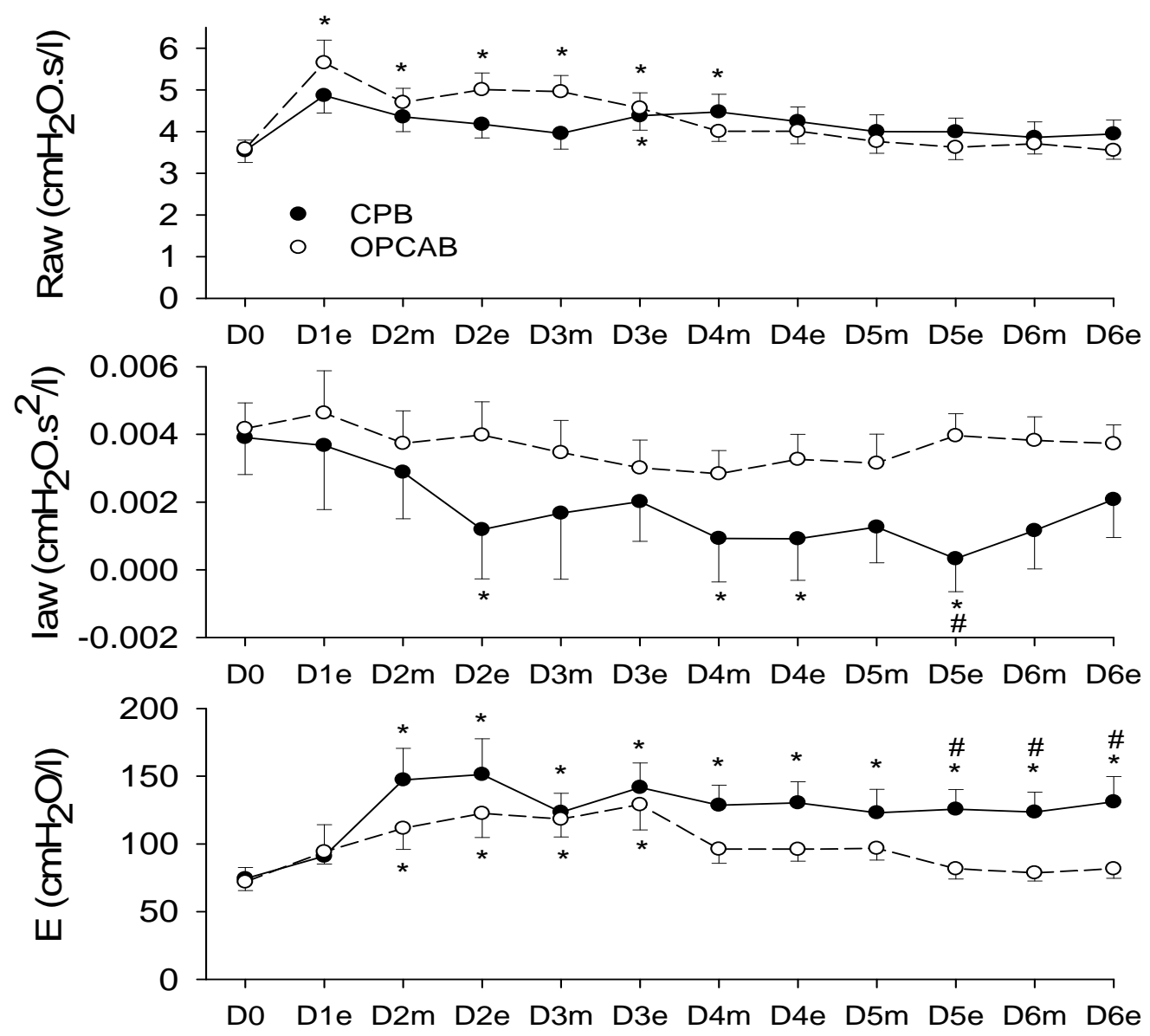

Figure 17. Mean values of airway resistance (Raw), inertance (Iaw), and respiratory elastance (E) before (D0) and after the cardiac surgery (D1e to D6e; $\mathrm{m}=$ morning; $\mathrm{e}=$ evening) in patients undergoing cardiopulmonary bypass $(\bullet)$ and off-pump coronary artery bypass $(\circ) .\left(^{*}: \mathrm{p}<0.05\right.$ versus the parameter values observed on D0; \#: $p<0.05$ between groups.)

The increase in Raw peaked a few hours after extubation and Raw subsequently gradually decreased, approaching closely the preoperative level by hospital discharge. There were only minor differences in the time course of Raw between the patients with or without CPB. Significant decreases were observed in Iaw for the patients with CPB $(p=0.009)$, whereas OPCAB surgery did not affect the postoperative changes of this parameter. The postoperative changes in $\mathrm{E}$ followed different patterns, with peak increases occurring later than those in Raw. The peak increase in $\mathrm{E}$ was somewhat higher in the patients with $\mathrm{CPB}$ and the elevation in $\mathrm{E}$ lasted longer than in the patients without $\mathrm{CPB}$, the differences proving significant at the last three assessments. The relative changes in Raw were fairly similar in the two groups of patients, whereas those in E were significantly greater at the end of the follow-up period (Fig. 18). 

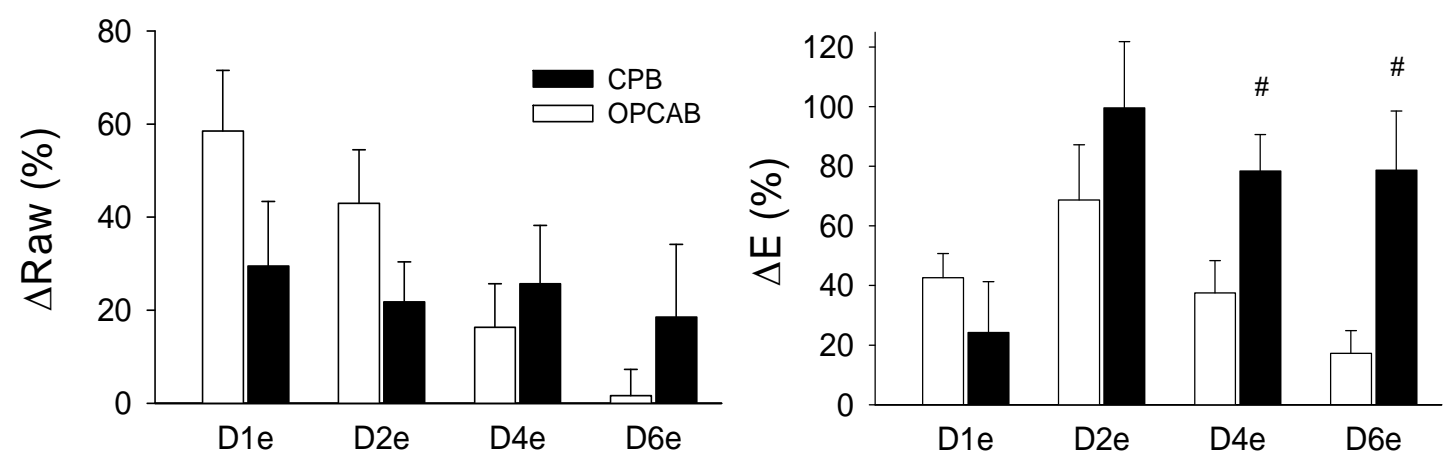

Figure 18. Changes in airway resistance ( $\Delta$ Raw) and respiratory elastance $(\Delta \mathrm{E})$ relative to the preoperative level observed during the evening of postoperative days 1 (D1e), 2 (D2e), 4 (D4e), and 6 (D6e) in patients who underwent cardiac surgery involving the use of a cardiopulmonary bypass (-) or an off-pump coronary artery bypass ( $\square$ ). (\#: $\mathrm{p}<0.05$ between groups.)

Influence of Obesity. Figure 19 depicts the time courses of the airway and tissue parameters in the patients classified as obese or not obese.
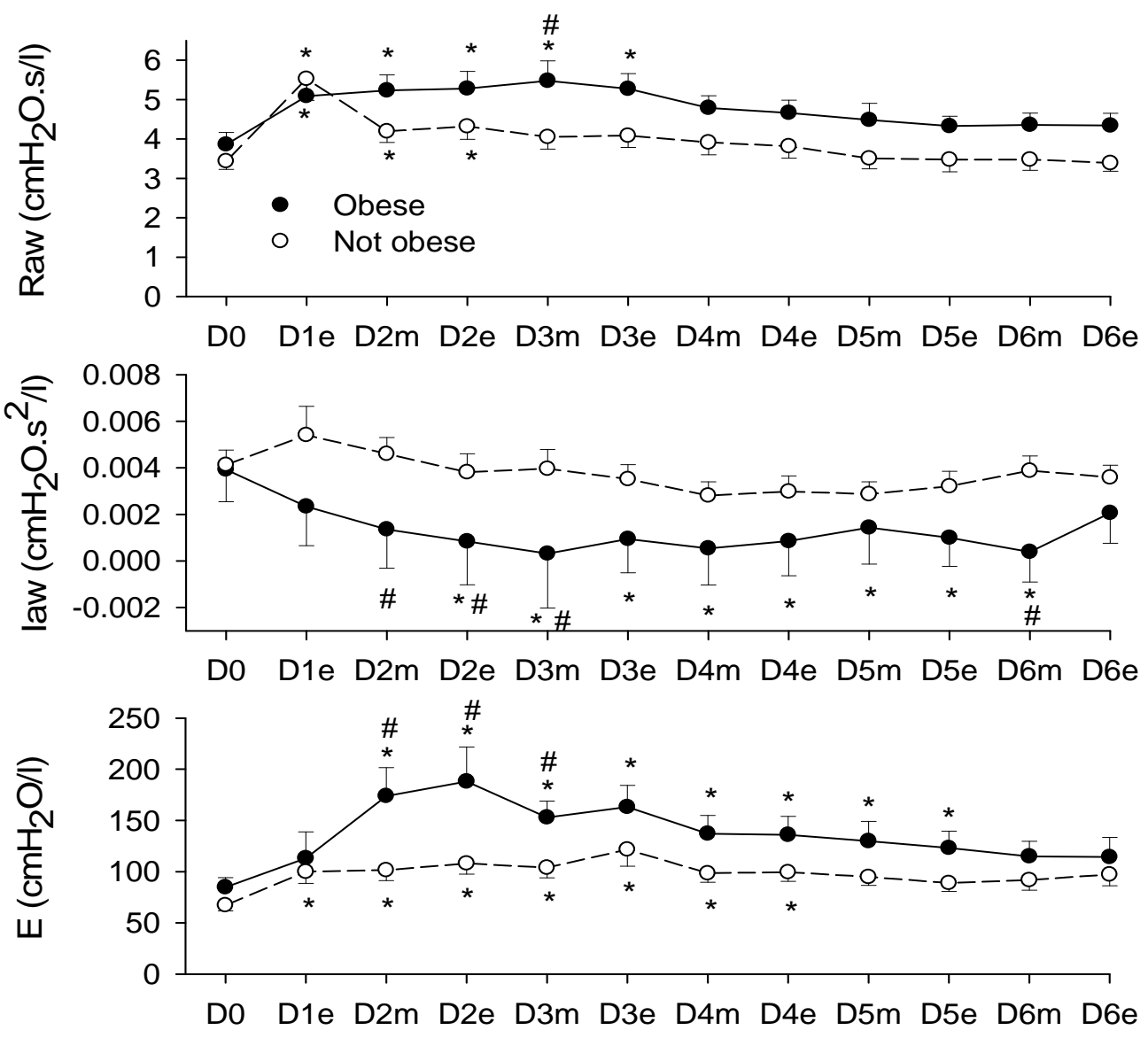

Figure 19. Lung mechanical properties (Raw, Iaw, E) before (D0) and after cardiac surgery (D1e to D6e; $m=$ morning; $\mathrm{e}=$ evening) in patients with $(\bullet)$ (body mass index $>30$,) or without ( $(\circ)$ obesity. $(*: \mathrm{p}<0.05$ versus the parameter values observed on D0; \#: $p<0.05$ between groups.) 
The magnitude of the peak postoperative increase in Raw was similar in the two groups, but its recovery was significantly more prolonged in the obese patients. The obese patients exhibited significant decreases in Iaw from days 2 to 6 , while Iaw remained at the initial level in the patients with OPCAB surgery. The peak increase in E was much more pronounced in the obese patients, and the preoperative level was reached by the end postoperative day 6 . These differences were reflected in the relative changes in the mechanical parameters (Fig. 20) with a significant difference in $\mathrm{E}(\Delta \mathrm{E})$ two days after surgery.
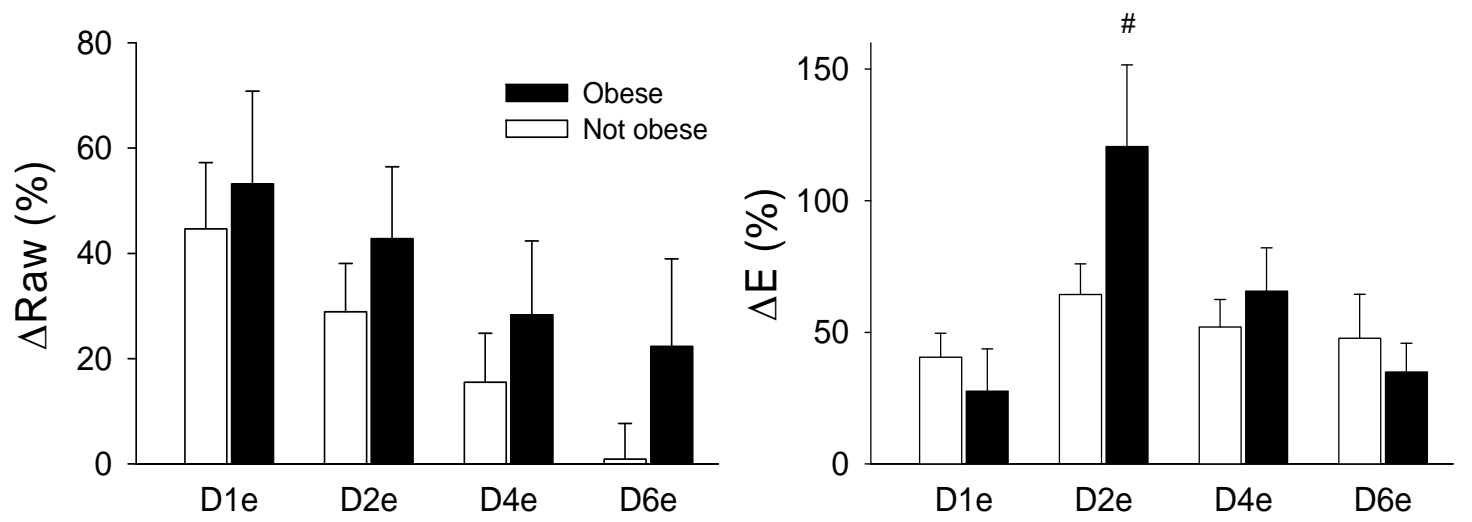

Figure 20. Changes in airway resistance $(\Delta \mathrm{Raw})$ and respiratory elastance $(\Delta \mathrm{E})$ relative to the preoperative level observed during the evening of postoperative days 1 (D1e), 2 (D2e), 4 (D4e), and 6 (D6e) in cardiac patients with ( $\square)$ (body mass index $>30$,) or without ( $\square$ ) obesity. (\#: p $<0.05$ between groups.)

\section{Discussion}

\subsection{The contribution of the pulmonary microvascular pressure in the maintenance of the alveolar architecture}

Artificial ventilation is often associated with deterioration in the lung mechanics and a loss of gas exchange capacity, with consequent hypoxemia. Therefore, precise characterization of all the contributing factors for the maintenance of the best possible lung mechanics is critical during mechanical ventilation, since the efficiency of this life support procedure is determined primarily by the surface available for gas exchange. The influence of the altered pressure in the pulmonary capillaries was systematically investigated on the airway and lung tissue parameters during mechanical ventilation at normal and lowered PEEP levels.

The results revealed that (1) mechanical ventilation at normal and low PEEP levels resulted in systematic changes in the lung mechanical parameters, as reflected by the Pcdependent elevations in the lung tissue mechanical parameters; (2) these adverse changes were inversely related to the level of the filling pressure in the pulmonary capillaries: the 
greatest increases in the parenchymal viscoelastic parameters were observed at $\mathrm{Pc}=0 \mathrm{mmHg}$, while maintenance of the physiological Pc led to significantly lower changes in these tissue parameters; and (3) the beneficial effects of the pressurized capillaries on the lung mechanics were also reflected in the shape of the $\mathrm{P}-\mathrm{V}$ curves: significant changes in the slope of the inflation limb and in the degree of hysteresis were observed during pulmonary hypoperfusion.

Nevertheless, maintenance of physiological hemodynamic conditions in the pulmonary circulation leads to the least compromised lung function, whereas a decrease of the pulmonary capillary filling results in marked adverse changes in the parenchymal mechanics. This phenomenon is even more pronounced when the PEEP level is lowered to below the closing volume. These findings suggest that the loss of alveolar stability due to the decreased level of capillary filling not only has an acute effect [2], but also precipitates the potentially deleterious consequences of mechanical ventilation. It is noteworthy, however, that a PEEP of $0.5 \mathrm{cmH}_{2} \mathrm{O}$ in isolated lungs corresponds to a lung volume below the normal closing volume. Therefore, it is likely that at the lower PEEP level, the observed influence of Pc on the lung mechanics are somewhat exaggerated. Nevertheless, under lung diseases associated with an increased potential of the lungs for closure, the beneficial effects of the maintenance of normal Pc may have significant role in the maintenance of an open lung.

The deleterious consequences of reductions in PEEP and Pc during mechanical ventilation are manifested mainly in the viscoelastic tissue parameters. It is noteworthy, however, that Raw exhibited slight, but significant increases after ventilation from a low PEEP, while Pc was maintained at 10 or $15 \mathrm{mmHg}$. This tendency to larger increases in Raw with increasing Pc may be attributed to the filling of the peripheral peribronchial vasculature that is supported by the pulmonary circulation, with a subsequent reduction in the overall bronchial diameter. The involvement of this mechanism is supported by the fact that a higher PEEP counteracted this effect by exerting efficient forces to stabilize the tracheobronchial tree $[44,45]$. Furthermore, application of the physiological PEEP in the presence of sufficient pulmonary vascular filling optimizes the alveolar architecture, leading to a less deleterious effect on the viscoelastic tissue parameters during mechanical ventilation. The current results demonstrate that the loss of tethering effects exerted by either the appropriate Ptp or the absence of pulmonary capillary filling results in a deterioration of the lung parenchymal mechanics. The mechanisms encountered during long-term ventilation involving shear stress, increased surface tension, and histological injuries [44] are not likely to play a role in the parenchymal mechanical deterioration observed in the present study, where the aim was the investigation of various Pc levels during relatively short ventilation times. 
Concerning the potential mechanism responsible for the elevations in the parenchymal mechanical parameters at a lowered pulmonary capillary pressure, it is noteworthy that $\mathrm{G}$ and $H$ raise proportionally resulting in a fairly constant $\eta$ (Fig. 2). This pattern of change suggests that the mechanism responsible for these findings is homogenous throughout in the lungs [46]. One possible interpretation of such results would be the derecruitment of lung units at low Pc levels, while the remaining communicating lung is homogenous and essentially unaltered. However, closures leading to $40-60 \%$ increases in $\mathrm{G}$ and $\mathrm{H}$ would have been reflected in a certain Pc-dependent elevations in Raw, which we did not observe. Alternatively, the influence of Pc on the viscoelastic properties of the parenchyma may be a consequence of homogenous structural changes in the connective tissue network. This observation is in full agreement with our previous findings demonstrating disorganization of the elastin fibers in the alveolar wall in the absence of pulmonary capillary filling [2].

The current results indicate that pressurizing the capillary network not only establishes a stable frame for the alveoli by optimizing the orientation of the elastin fibers [2], but also prevents alveolar collapse during mechanical ventilation. The loss of stability in the lung periphery, particularly when the low PEEP was associated with a low Pc, is reflected in the distortion of the $\mathrm{P}-\mathrm{V}$ curves demonstrating increases in their slope and hysteresis (Fig. 5). The establishment of lung expansion through maintenance of the physiological PEEP and perfusion of the pulmonary vasculature with normal filling pressures results in the least harmful changes in the oscillatory mechanical parameters and in the $\mathrm{P}-\mathrm{V}$ curve indices. Moreover, expansion of the lungs following recruitment maneuvers reversed the increases in the airway and tissue parameters, suggesting that the normal geometry in the lung periphery is partially re-established, most likely via realignment of elastin fibers.

In summary, our results demonstrate that, during mechanical ventilation, the filling of the pulmonary capillaries plays an important role in keeping the lung open. A reduction in the expanding pressure in the airways or in the pulmonary capillaries leads to marked acute deteriorations in the lung mechanics, with predominant changes in the damping and elastic properties of the parenchyma. Thus, optimization of the pulmonary hemodynamics should be paired with the ventilation strategy in order to establish the most favorable lung function. As an important mechanical factor in the maintenance of the stability of the lung periphery, the physiological pressure in the pulmonary capillaries should be taken into account, especially in situations where the pulmonary capillary perfusion may be impaired (e.g. in hypovolemia, pulmonary hypotension, or lung embolism) during mechanical ventilation. 


\subsection{Mechanical and vascular changes in the lungs during inflation- defleation maneuvers generated by positive and negative pressures}

Simultaneous changes in lung mechanical and pulmonary vascular parameters during PPLI and NPLI were related in the present study. PPLI and NPLI generated similar courses in the airway and parenchymal mechanical parameters, with decreases in Raw and marked increases in the viscous and elastic parameters of the lung parenchyma. In contrast, changes in Rv were influenced considerably by the mode of lung inflation: increases were observed during PPLI, whereas NPLI caused mild decreases in Rv. The parenchymal parameters displayed hysteresis with the lung inflation pressure, independently of the mode of inflation, resulting in lower $G$ and $\mathrm{H}$ values in the expiratory phase. Conversely, hysteresis for $\mathrm{Rv}$ was observed only during PPLI, where this parameter was significantly greater during the same pressures at expirations.

Methodological considerations. An important feature of the ventilation perfusion circuit applied in the present study is that the reservoirs were placed outside the box containing the lung. Thus vascular pressures were kept constant relative to the pleural pressure during PPLI and relative to the alveolar pressure (Palv) during NPLI. The opposite changes in Rv between PPLI and NPLI evidence that the transmural pressure differences in the pulmonary vasculature were indeed different in the two modes of inflations, because the blood reservoirs supporting the pulmonary artery and collecting blood from the pulmonary vein have been kept on a common pressure level during the different inflation maneuvers. In the experimental model, the standardization of the perfusion pressures avoids the confounding influences due to the altered cardiac output induced by the different inflation maneuvers in an intact chest. Therefore, the present findings highlight the primary importance of the pressure differences both across the airway tree and the pulmonary vasculature, while the absolute pressure level in these compartments per se does not matter: the NPLI and PPLI maneuvers lead to similar pressure gradient through the tracheobronchial tree, and opposing transmural pressure gradients. It has been established that the interpretation of the changes in the pulmonary vascular mechanics with altered lung volume is fundamentally dependent on the manner by which the vascular pressure is related to different reference pressures (i.e., pleural or alveolar) [47]. In the present study, we standardized our pressure regimen by maintaining constant Ppa and Pla with reference to atmosphere during the PPLI and NPLI maneuvers. This approach ignores the possible changes in the perfusion pressure that may occur in a closed chest during lung expansions with negative pressure around the chest, and thus extrapolation of the present results to an in vivo condition is not straightforward. 
Effects of lung inflations on lung mechanics. As described previously [20, 21, 48], the decreases in Raw reflect pressure-dependent increases in the airway caliber, while the increases in the parenchymal mechanical parameters are related to the increased damping and stiffness of the lung tissue at high inflation pressures. Our results revealed that the mode of inflation does not affect the pressure-dependent changes in the airway and parenchymal mechanics, as an indication that the forces determining the airway caliber and changes in viscous properties and elastic recoil of the lung are independent of the manner in which Ptp is maintained. The pressure gradient between the alveoli and the airway opening is similar for the two maneuvers, merely the absolute values of the airway opening and hence Palv being greater during PPLI, which may result in a decrease of a few percent in the alveolar gas compliance $(\mathrm{Cg})$. Because the effect of $\mathrm{Cg}$ is negligible relative to that of the lung tissue compliance, small changes in $\mathrm{Cg}$ are unlikely to be detected, which explains the lack of difference between the mechanical effects on NPLI and PPLI.

Effects of PPLI on $R v$. In agreement with the results of previous studies [12, 13, 15-18, 22], we observed monotonic increases in Rv during PPLI maneuvers. The underlying physiological phenomena have been well established and may involve the compression of the intra-alveolar vessels by the increased positive pressure leading to compression and distortion of the capillaries, in addition to the elongation of intra- and extra-alveolar vessels [12-17]. These mechanisms seem to predominate in the changes in $\mathrm{Rv}$ in the present study, whereas the enlargement of extra-alveolar vascular compartments $[13,14,16]$ is not reflected in its net changes. Although the changes in Rv with lung inflation or deflation have been extensively investigated previously, the effects of cyclic changes in PPLI (as occurs during mechanical ventilation) have been addressed in only a few investigations [16, 17, 22]. In the present study, Rv was consistently higher during deflations than during inflations at isopressure points, and this hysteresis diminished when the changes in Rv were plotted against lung volume. Although this finding is consistent with the results of previous investigations $[16,17$, 22], the mechanisms responsible for this pattern of change have not been clarified. Studies of individual pulmonary vessels by using morphological [49] or mechanical approaches [50] suggest that pulmonary vascular volumes are greater during lung deflations, which seemingly contradicts the present and previous findings obtained for the whole lung. This apparent controversy can be explained by the possibility that the intraalveolar capillaries collapse in consequence of a PPLI that compresses the alveolar walls [12-17]. If this pulmonary capillary derecruitment occurs during PPLI, Rv at isopressure points remains higher for the inspiratory limb because, during expiration, the pulmonary capillaries regain their patency when $\mathrm{Pc}$ 
overcomes the compression pressure exerted by the positive intra-alveolar pressure. This phenomenon may be a consequence of approaching West zone 1 condition by the end of positive-pressure inflation (Palv $>\mathrm{Ppa}>\mathrm{Pla}$ ) where capillaries collapse. To provide experimental evidence for the presence of this mechanism, we performed additional experiments in five further lungs where PPLI maneuvers were repeated at a physiological $\left(\mathrm{Pc}=13.6 \mathrm{cmH}_{2} \mathrm{O}\right)$ and at an elevated $\mathrm{Pc}$ of $20.4 \mathrm{cmH}_{2} \mathrm{O}$. In these experiments we found that elevation of $\mathrm{Pc}$ to $20.4 \mathrm{cmH}_{2} \mathrm{O}$ (West zone 2 and 3 conditions) led to a marked reduction in the hysteresis, suggesting that closure of the intra-alveolar capillaries plays a role in the elevated $\mathrm{Rv}$ values obtained in the main study population during expiration.

Effects of NPLI on Rv. In accordance with the results of a previous study by Burton and Patel [18], we observed mild, but statistically significant decreases in Rv during the NPLI maneuvers. Other authors, however, have reported decreases in Rv at low levels of Ptp, followed by an increase in Rv at high pressures, when lung expansions were generated by a vacuum around excised dog lungs $[13,23]$ and in open-chest dogs [15]. Although the reason for this discrepancy in the literature is not clear, the difference in the position of the perfusion reservoirs relative to the ventilation apparatus or the dynamics of lung inflation (steady-state vs. dynamic) may be involved. In the present study, the reservoirs were kept at atmospheric pressure, and thus perfusion pressures were not submitted to the changes in the Ptp. The importance of the positioning of the perfusion reservoirs during NPLI has been highlighted by Permutt et al. [47], who concluded that the differences observed in the changes in Rv during PPLI and NPLI disappear when the perfusion pressures were kept constant relative to Ptp. Moreover, in the present study we generated slow lung inflations rather than elevating Ptp stepwise. Maintaining Ptp elevated for a longer period may have biased the influence of lung inflation on $\mathrm{Rv}$ through activation of the autonomous nervous system in the in vivo experiments [15]. The present experimental setting allows clarification of the mechanisms responsible for the decrease in Rv during NPLI with constant perfusion pressures, because only the mechanical effect of Ptp on the pulmonary vasculature is involved, all other confounding factors are excluded. Thus it seems plausible that NPLI exerts radial traction on the capillaries in the walls of the subpleural alveoli, and increasing their crosssectional area.

Interdependence between lung mechanics and pulmonary vasculature. Although numerous previous studies investigated separately the effects of different modes of inflation on the lung vasculature [12, 13, 15-18, 22, 23] or on the lung mechanics [19-21], this is the first report studying simultaneous changes during PPLI and NPLI maneuvers. The effects of the parenchyma on the mechanical status of the capillaries are rather trivial, because the 
transmission of Ptp to the vascular wall during breathing is determined by the local and overall viscoelastic properties. As regards the other direction of the interdependence, there is evidence that filled pulmonary capillaries exert a mechanical tethering force to maintain the normal alveolar geometry and hence lung compliance [2, 51]. Because the filling of the alveolar capillaries is more promoted during NPLI maneuvers, this phenomenon may be expected to result in lower values of lung tissue parameters during these maneuvers. The fact that we did not observe significant differences in the mechanical parameters between the PPLI and NPLI maneuvers (Fig. 9) suggests that this phenomenon did not play a role in the range of Ptp levels used in the present study. During PPLI maneuvers, the pulmonary capillaries tend to empty only at high Ptp levels, where Ptp alone determines the alveolar geometry [2].

Summary and implications. We have demonstrated that opposing modes of inflation (PPLI, NPLI) resulted the same lung mechanical condition, but recruitment maneuvers with positive airway pressure may jeopardize the pulmonary hemodynamic conditions by derecruiting the pulmonary capillaries and subsequently affecting the afterload of the right ventricle. The adverse changes observed in Rv were fairly small; however, they may have a greater impact at the higher Ptp levels often reached during positive-pressure mechanical ventilation, especially in clinical conditions where pulmonary hypertension is already present (e.g., congenital heart disease, acute lung injury or chronic pulmonary diseases). Extrapolation of the present findings to a physiological condition is prohibited by the fact that the perfusion pressures during NPLI remained constant. Nevertheless, the decrease in Rv observed in the present study during NPLI may be beneficial under particular clinical conditions where the lungs can be ventilated with negative pleural pressure and where the perfusion is independent of the Ptp regimen (e.g., extracorporeal membrane oxygenation or right ventricular assistance).

\subsection{Mechanisms of bronchial hyper-responsiveness following coronary ischemia}

The separate assessment of the airways and the respiratory tissue mechanics in the present study has revealed that the basal mechanical properties of these major lung compartments are not affected by chronic pulmonary congestion following left-ventricular failure. Nevertheless, it provided evidence of the presence of lung hyperresponsiveness to constrictor stimuli following left-heart failure. Investigation of the underlying pathophysiological mechanisms responsible for these phenomena demonstrated that (1) in the presence of myocardial ischemia (MI), the development of BHR is independent of the level of the EDLVP; (2) a 
chronically elevated EDLVP causes remodeling in the pulmonary vasculature; (3) sustained post-capillary pulmonary hypertension leads to a remodeling in the bronchial and alveolar wall with the proliferation of pericytes stained for $\alpha$-SMA antibody.

Lung congestion was achieved by inducing left-ventricular myocardial ischemia. This experimental model has been proved to provide post-capillary pulmonary hypertension leading to chronic lung congestion [52] with a high survival rate [53]. Further, the magnitude of the myocardial necrosis was sufficient to elevate EDLVP (more than twofold), confirming the impairment in the left-ventricular compliance, hence an increase in the pulmonary venous pressure. The complete returns in the mechanical parameters following serotonin challenges independent from the presence or absence of coronary ischemia implies that the changes we measured resulted from the chronic lung congestion, rather than the acute effects of serotonin.

Although acute changes in the pulmonary hemodynamic conditions have often been demonstrated to influence the mechanical properties of the respiratory system $[1,54,55]$, we did not observe statistically significant changes in the baseline values of the respiratory mechanical parameters following a sustained elevation in left-atrial pressure. This finding is in agreement with previous observations that chronic elevations in pulmonary vascular pressures have no consistent consequences on the central airway resistance $[56,57]$, where the edema affects primarily the pulmonary vasculature and is not severe enough to be manifested in the appearance of extravascular edema fluid around the bronchial wall [54]. Reports of impairments in the lung mechanical parameters mentioned the presence of severe interstitial and peribronchial edema $[58,59]$ which was not the case in the present study.

Despite the normal baseline respiratory mechanics observed after the induction of pulmonary vascular engorgement, left-heart failure led to a significant elevation in the lung responsiveness to a constrictor stimulus. Serotonin causes smooth muscle contraction by 5 HT2 receptor activation and by stimulating 5-HT1A receptors that further potentiates its effects on 5-HT2 receptors [60]. In addition, serotonin increases release of acetylcholine thereby enhancing the cholinergic tone of the airway smooth muscle. Previous studies on patients with left-heart failure yielded inconsistent conclusions regarding the presence or absence of BHR, with a majority of the papers reporting airway hyper-responsiveness to exogenous cholinergic constrictor stimuli $[3-5,8]$, whereas others described a normal airway reactivity $[24,25]$. The previous studies, however,were conducted under clinical settings on a wide variety of patients as concerns the type, the severity and the history of the respiratory and circulatory disease, factors which may bias an evaluation of the lung responsiveness. Our study, conducted under well-controlled conditions, confirms the occurrence of BHR in the 
presence of left-heart failure $[3-5,8]$, with the additional information that the airways display an enhanced reactivity to a constrictor agonist (serotonin) that is not acting on the cholinergic receptors directly. The changes in the Raw following serotonin challenges demonstrate the presence of a dose-dependent airway constriction, while the increases in $\mathrm{G}$ exceeding those in $\mathrm{H}$ are likely to be a consequence of severe ventilation heterogeneities [46].

The mechanism responsible for the development of BHR during chronic lung congestion is far from clear. Some authors have suggested that, after the administration of cholinergic agonists with vasodilator properties, bronchial vasodilation plays a major role by distorting the airway geometry $[3,8,61]$. However, we observed BHR to a bronchoactive agent with a pulmonary vasoconstrictor potential, thus this mechanism was not involved in the enhanced responsiveness of the airways. Moreover, in line with previous findings [5], we found no relationship between the degree of lung congestion estimated from EDLVP (Fig. 12) and the severity of BHR. Although mucosal swelling, peribronchial edema and airway smooth muscle hypertrophy have also been proposed as mechanisms contributing to BHR during pulmonary hypertension $[3,26,27,54,62]$, we were unable to detect any of these structural changes in the bronchial wall after left heart failure while BHR was still present. This finding suggests that uncoupling of the airways from the lung parenchyma [29] was not involved either. The serotonin-induced changes in the systemic blood pressures were not affected by the presence of coronary ischemia. Therefore, enhancements in the tracheal smooth muscle tone due to greater increases in systemic blood pressure following [63] MI did not play a role in the development of BHR. Besides altered vasculature, changes in airway wall geometry observed during acute vascular engorgement have been demonstrated to induce BHR $[8,64]$. Since thickening of the airway wall following MI was also observed in the present study, geometrical changes in the bronchial walls may have contributed to the development of BHR.

It is noteworthy that the histological preparations displayed proliferation of cells expressing $\alpha$-SMA. This indicates an increase in the number of pericytes (both in the alveolar septa and around the airways) in lungs exposed chronically to a high pulmonary vascular pressure (Figs. 13 and 14). This finding is in agreement with earlier results demonstrating a lung structural remodeling associated with the proliferation of pericytes and the differentiation of fibroblasts into contractile myofibroblasts during congestive heart failure $[52,65,66]$. Myofibroblasts have been shown to play an important role not only in lung parenchymal remodeling, but also in enhancing bronchoconstriction through the expression of contractile proteins [65]. Thus, our results suggest that the enhanced expression of $\alpha$-SMA plays an important role in the development of BHR during sustained lung congestion. 
Elevated pulmonary vascular pressures have been demonstrated to stimulate unmyelinated C-fiber nerve endings in the airways and in the lung tissue $[67,68]$. The activation of these fibers could also play a role in the development of airway narrowing [69]. Since serotonin has been reported to potentiate the excitatory effects of theses fibers [70, 71], further investigations are needed to clarify the role of this neuronal pathway.

In conclusion, in an attempt to clarify the pathophysiological mechanisms responsible for the enhanced airway responsiveness to serotonin, we found that BHR during pulmonary vascular engorgement is most probably due to the expression of $\alpha$-SMA in the lungs rather than a consequence of the interaction of the pulmonary vasculature with the bronchial tree. These findings imply that processes leading to BHR subsequent to left-ventricular failure resemble those involved in allergic asthma. The clinical terminology "cardiac asthma" used in patients with left-heart failure associated with respiratory symptoms therefore makes sense.

\subsection{Changes in the mechanics of the respiratory system after cardiac surgery}

In the present study the changes in the airway and respiratory tissue mechanics were followed during the critical first week after cardiac surgery, the period when most of the respiratory complications occur. Forced oscillations technique proved to be appropriate for measurements of the respiratory mechanics in this patient population, whose ability to cooperate in the postoperative period was limited. Impedance data showed development of airway narrowing immediately after extubation, which slowly diminished up to the time of hospital discharge. By contrast, the peak deterioration in the elastic properties of tissues was observed around the $2^{\text {nd }}$ postoperative day. Gender, smoking history, the age of the patients, and the duration of the surgery had no impact on the postoperative changes in the respiratory function, whereas the inclusion of $\mathrm{CPB}$ in the cardiac surgery prolonged the postoperative deterioration in the respiratory system elastance. The presence of obesity, on the other hand, elongated the duration of postoperative airway narrowing and enhanced the peak elevation in the elastance.

The time courses of the changes in the airway and respiratory tissue parameters were dissociated in the postoperative period, which suggests that the mechanisms responsible for these changes are fundamentally different (Fig. 16). While the presence of positive total fluid balance might have contributed to the immediate postoperative airway narrowing, previous studies [72] have demonstrated upper airway narrowing after extubation at a proximal site of the conducting airways. Because Raw reflects primarily the flow resistance of the upper 
airways, the postextubation increases in this parameter most probably reflect this phenomenon. Our follow-up data revealed that the airway narrowing subsequent to the irritation caused by the endotracheal tube persists for about four days after extubation, and a complete recovery can generally be expected around the $5^{\text {th }}$ postoperative day. Considering that obesity has proved to be an important factor associated with impaired upper airway patency [73], the presence of prolonged airway narrowing in our obese patients (Figs. 19; 20) is in line with the primary involvement of the upper airways in the Raw increases. The relatively neutral role of CPB in the time course of Raw (Figs. 17; 18) further substantiates the involvement of the upper airway irritation, as this intervention is expected to exert its adverse effects at the lung periphery [36, 74-80].

The increase in E after cardiac surgery reflects the stiffening of the respiratory tissues. The pulmonary and chest wall contributions to $\mathrm{E}$ have been reported to be approximately equal in adult humans [81]. Although E combines the mechanical properties of the lungs and the chest wall, the fact that the chest wall elastance was earlier found to be unchanged after cardiac surgery [81] suggests that the lungs are responsible for the changes observed in the present study. The increased stiffness of the respiratory tissues could be subsequent to an alteration in elasticity of the lung parenchyma itself by systemic inflammatory response syndrome [82] leading to edema development [76]. However, these syndromes together with excessive mucus secretion, pain, upward shift of diaphragm into the thoracic compartment, pneumothorax, and pleural effusion all result in atelectasis development and lung volume loss $[74,75]$, which may be the main factor responsible for the elevated E. The highly probable presence of restrictive processes in our subjects in the postoperative period is confirmed by our radiographic findings and the consistency with results of previous studies [32, 35, 78, 83] demonstrating atelectasis with a decrease in the functional residual capacity toward the closing capacity after cardiac surgery. As the presence of small airway closures with subsequent development of ventilation heterogeneities has been shown to decrease the Iaw [38], the falls in Iaw in the CPB and obese patients further indicates the development of restrictive processes in the lung periphery. The shallow breathing of the patients after surgery was not sufficient to induce alveolar recruitment. Obesity is a major risk factor for the development of postoperative atelectasis [84], and the major role of this phenomenon is confirmed by the significantly greater postoperative increase in $\mathrm{E}$ in the obese patients.

As concerns the mechanism responsible for the higher postoperative rise in $\mathrm{E}$ in the $\mathrm{CPB}$ patients, the injury induced by temporary total lung ischemia-reperfusion has been demonstrated to lead to damage to the pneumocyte II cells in the alveolar walls [79]. This 
damage results in a decreased or even interrupted surfactant production, which promotes the development of atelectasis and the loss of alveolar surface available for gas exchange [79]. In agreement with previous findings where the gas exchange parameters [33] or functional residual capacity [35] were measured, the tissue mechanical parameters peaked around two days after the surgery and exhibited gradual improvements thereafter. As the surfactant halflife of 15 to 36 hours lies within this period [85], this time course supports the involvement of the diminished surfactant function in the deterioration of the postoperative lung function. This surfactant damage takes time to evolve, which explains why the effect of CPB was not apparent in the postoperative lung function immediately after chest closure [86, 87], and which verifies the primary importance of the first week in the follow-up of the lung function changes after cardiac surgery. The total positive fluid balance was more positive in the CPB patients with a mean difference of $555 \mathrm{~mL}(\mathrm{p}=0.057)$. If this excess of fluid had an influence on the postoperative lung mechanics, it should have been manifested in immediate postoperative elevations of E. Because the $\mathrm{E}$ values were comparable between the $\mathrm{CPB}$ and OPCAB patients immediately after extubation (D1e, Fig. 17), the difference in the fluid balance did not likely contribute to the greater increases in $\mathrm{E}$ in the patients with CPB.

In summary, the present study has demonstrated the ability of the forced oscillation technique to follow the changes in the airway and respiratory tissue mechanics in patients in the critically important period after cardiac surgery, when the postoperative respiratory morbidity is most significant. The immediate airway narrowing uniformly observed in our patients after extubation may be a consequence of the mechanical irritation exerted by the endotracheal tube in the upper airways, which normalized by five to six days postoperatively. The increases in E, which peaked on the postoperative day 2 or 3 , may indicate the development of atelectasis, as a result of the mechanical ventilation, the prolonged supine position, and the restricted respiratory movements. Because obesity exaggerates and prolongs these adverse effects, obese patients exhibited more severe postoperative respiratory complications two to five days after cardiac surgery. The more extensive and longer stiffening of the respiratory tissues in the $\mathrm{CPB}$ patients may be attributed to the prior decrease in the production of surfactant, as a result of the temporary pulmonary ischemia during CPB. These results demonstrate the need for particular attention in the postoperative management of patients after cardiac surgery in order to reduce the immediate deterioration of the airway function, and to take steps to avoid atelectasis development during the critical postoperative days 2 and 3. These measures have particular importance in the maintenance of the best possible lung function in obese patients and (or) if the surgery involves the use of CPB. 


\section{Summary and Conclusions}

In all studies included in the present thesis, the mechanical properties of the lungs or the respiratory system were measured by the forced oscillation technique either via the wave tube technique or by applying forced oscillations around the spontaneous breathing frequency. Separation of the airway and parenchymal components revealed the following findings:

- During mechanical ventilation, the filling of the pulmonary capillaries plays an important role in the maintenance of the stability of the alveolar architecture and reduction in the expanding pressure in the airways or in the pulmonary capillaries leads to an acute deteriorations in the lung mechanics. Therefore in order to establish the most favorable lung function during mechanical ventillation, ventilation strategy should be paired with the optimization of the pulmonary hemodynamics, especially in situations where the pulmonary capillary perfusion may be impaired.

- Recruitment maneuvers with positive airway pressure may jeopardize the pulmonary hemodynamic conditions by derecruiting the pulmonary capillaries and subsequently affecting the afterload of the right ventricle. Considering the fact that in our study the perfusion pressures during negative pressure lung inflations remained constant, the decrease in the vascular resistance during these maneuvers may be beneficial under particular clinical conditions where the lungs can be ventilated with negative pleural pressure and where the perfusion is independent of the Ptp regimen.

- Chronic elevation of left atrial pressure after left heart failure leads to bronchial hyperresponsiveness without affecting the baseline airway or tissue mechanical properties. The observed airway hyper-responsiveness during pulmonary vascular engorgement is most probably due to the expression of $\alpha$-SMA cells in the lungs rather than a consequence of the interaction of the pulmonary vasculature with the bronchial tree.

- We demonstrated the ability of the forced oscillation technique to follow the changes in the airway and respiratory tissue mechanics in patients after cardiac surgery in a critically important period, when most of the respiratory complications occur and the postoperative respiratory morbidity is most significant. The impedance data revealed the development of airway narrowing immediately after extubation and the peak deterioration in the elastic properties of the respiratory tissues around the $2^{\text {nd }}$ postoperative day. Our results demonstrate the need for particular attention in the postoperative management of patients after cardiac surgery in order to reduce the immediate deterioration of the airway function, and to take steps to avoid atelectasis 
development during the critical postoperative days. These measures have particular importance in the maintenance of the best possible lung function in obese patients and (or) if the surgery involves the use of CPB.

The studies included in this thesis may help to better understand the relationships between the pulmonary vasculature and the complex structure of the bronchoalveolar network and to better understand the cardiopulmonary interactions between the different compartments of lung mechanics and pulmonary hemodynamics. Although it is difficult to extrapolate experimental findings to clinical practice, the results of the present thesis may lead to an improvement in the anaesthesia management of patients with at risk of lung function deterioration and impaired pulmonary hemodynamics.

\section{Acknowledgements}

First and foremost, I would like to acknowledge the support of my two supervisors.

I am grateful to my supervisor Dr Ferenc Peták for all the teachings and for his invaluable help in conducting most of the experimental work, data analysis and the preparation of the thesis. I greatly appreciate his guidance and his excellent advices.

My most grateful thanks and appreciation to my supervisor Professor Walid Habre who supported me so strongly during all these years. I also greatly appreciate his continuous guidance and invaluable advices, the trust, independence and all the opportunities he has afforded me.

I would like to express my thanks to Professor Zoltán Hantos who supported me strongly during all the steps of my research career. I am honoured to have worked under his supervision, and especially thank him for his invaluable guidance and instructions and for all the opportunities he has afforded me.

I am also very grateful to Dr. Barna Babik who introduced me to this research area and for his continuous guidance and support. His excellent advices have been essential in the development of my research career.

I also wish to express my thanks to Professor Denis Morel and all the staff of the Anaesthesiological Investigation Unit at the University of Geneva for their contribution througout the years, especially to Fabienne, Manu and Sylvie.

I must not forget to thank Anne for her continuous support in the most difficult days. Merci!

The work presented in this thesis was supported by the Hungarian Scientific Research Grant (OTKA F38340; T62403; K62403) and the Swiss National Science Foundation Grant (No. 3200-105828; 3200-105828/1). 


\section{References}

1. Petak, F., W. Habre, Z. Hantos, P.D. Sly, and D.R. Morel. Effects of pulmonary vascular pressures and flow on airway and parenchymal mechanics in isolated rat lungs. J Appl Physiol, 2002. 92(1): p. 169-78.

2. Petak, F., B. Babik, Z. Hantos, D.R. Morel, J.C. Pache, C. Biton, B. Suki, and W. Habre. Impact of microvascular circulation on peripheral lung stability. Am J Physiol Lung Cell Mol Physiol, 2004. 287(4): p. L879-89.

3. Cabanes, L.R., S.N. Weber, R. Matran, J. Regnard, M.O. Richard, M.E. Degeorges, and A. Lockhart. Bronchial hyperresponsiveness to methacholine in patients with impaired left ventricular function. $N$ Engl J Med, 1989. 320(20): p. 1317-22.

4. Pison, C., J.L. Malo, J.L. Rouleau, J. Chalaoui, H. Ghezzo, and J. Malo. Bronchial hyperresponsiveness to inhaled methacholine in subjects with chronic left heart failure at a time of exacerbation and after increasing diuretic therapy. Chest, 1989. 96(2): p. 230-5.

5. Sasaki, F., T. Ishizaki, J. Mifune, M. Fujimura, S. Nishioka, and S. Miyabo. Bronchial hyperresponsiveness in patients with chronic congestive heart failure. Chest, 1990. 97(3): p. 534-8.

6. Lanteri, C.J., S. Kano, A.W. Duncan, and P.D. Sly. Changes in respiratory mechanics in children undergoing cardiopulmonary bypass. Am J Respir Crit Care Med, 1995. 152(6 Pt 1): p. 1893-900.

7. Habre, W., N. Schutz, M. Pellegrini, M. Beghetti, P.D. Sly, Z. Hantos, and F. Petak. Preoperative pulmonary hemodynamics determines changes in airway and tissue mechanics following surgical repair of congenital heart diseases. Pediatr Pulmonol, 2004. 38(6): p. 470-6.

8. Rolla, G., C. Bucca, E. Caria, E. Scappaticci, and S. Baldi. Bronchial responsiveness in patients with mitral valve disease. Eur Respir J, 1990. 3(2): p. 127-31.

9. Lubica, H. Pathologic lung function in children and adolescents with congenital heart defects. Pediatr Cardiol, 1996. 17(5): p. 314-5.

10. Tremblay, L.N., T. Yamashiro, K.N. DeCampos, B.V. Mestrinho, A.S. Slutsky, T.R. Todd, and S.H. Keshavjee. Effect of hypotension preceding death on the function of lungs from donors with nonbeating hearts. J Heart Lung Transplant, 1996. 15(3): p. 260-8.

11. Mitzner, W., W. Lee, D. Georgakopoulos, and E. Wagner. Angiogenesis in the mouse lung. Am J Pathol, 2000. 157(1): p. 93-101.

12. Dawson, C.A., D.J. Grimm, and J.H. Linehan. Effects of lung inflation on longitudinal distribution of pulmonary vascular resistance. J Appl Physiol, 1977. 43(6): p. 1089-92.

13. Hakim, T.S., R.P. Michel, and H.K. Chang. Effect of lung inflation on pulmonary vascular resistance by arterial and venous occlusion. J Appl Physiol, 1982. 53(5): p. 1110-5.

14. Howell, J.B., S. Permutt, D.F. Proctor, and R.L. Riley. Effect of inflation of the lung on different parts of pulmonary vascular bed. J Appl Physiol, 1961. 16: p. 71-6.

15. Roos, A., L.J. Thomas, Jr., E.L. Nagel, and D.C. Prommas. Pulmonary vascular resistance as determined by lung inflation and vascular pressures. J Appl Physiol, 1961. 16: p. 77-84.

16. Sun, R.Y., G.F. Nieman, T.S. Hakim, and K.K. Chang. [Effects of lung volume and alveolar surface tension on pulmonary vascular resistance]. Zhongguo Yi Xue Ke Xue Yuan Xue Bao, 1987. 9(6): p. 439-4. 
17. Whittenberger, J.L., G.M. Mc, E. Berglund, and H.G. Borst. Influence of state of inflation of the lung on pulmonary vascular resistance. J Appl Physiol, 1960. 15: p. 878-82.

18. Burton, A.C. and D.J. Patel. Effect on pulmonary vascular resistance of inflation of the rabbit lungs. J Appl Physiol, 1958. 12(2): p. 239-46.

19. Gomes, R.F., X. Shen, R. Ramchandani, R.S. Tepper, and J.H. Bates. Comparative respiratory system mechanics in rodents. J Appl Physiol, 2000. 89(3): p. 908-16.

20. Hantos, Z., R.A. Collins, D.J. Turner, T.Z. Janosi, and P.D. Sly. Tracking of airway and tissue mechanics during TLC maneuvers in mice. J Appl Physiol, 2003. 95(4): p. 1695-705.

21. Hirai, T., K.A. McKeown, R.F. Gomes, and J.H. Bates. Effects of lung volume on lung and chest wall mechanics in rats. J Appl Physiol, 1999. 86(1): p. 16-21.

22. Creamer, K.M., L.L. McCloud, L.E. Fisher, and I.C. Ehrhart. Ventilation above closing volume reduces pulmonary vascular resistance hysteresis. Am J Respir Crit Care Med, 1998. 158(4): p. 1114-9.

23. Thomas, L.J., Jr., Z.J. Griffo, and A. Roos. Effect of negative-pressure inflation of the lung on pulmonary vascular resistance. J Appl Physiol, 1961. 16: p. 451-6.

24. Eichacker, P.Q., M.J. Seidelman, M.S. Rothstein, and T. Lejemtel. Methacholine bronchial reactivity testing in patients with chronic congestive heart failure. Chest, 1988. 93(2): p. 336-8.

25. Seibert, A.F., R.C. Allison, C.H. Bryars, and M.B. Kirkpatrick. Normal airway responsiveness to methacholine in cardiac asthma. Am Rev Respir Dis, 1989. 140(6): p. 1805-6.

26. Okazawa, M., N. Muller, A.E. McNamara, S. Child, L. Verburgt, and P.D. Pare. Human airway narrowing measured using high resolution computed tomography. Am J Respir Crit Care Med, 1996. 154(5): p. 1557-62.

27. Brown, R.H., E.A. Zerhouni, and W. Mitzner. Airway edema potentiates airway reactivity. J Appl Physiol, 1995. 79(4): p. 1242-8.

28. Fishman, A.P. Cardiac asthma--a fresh look at an old wheeze. N Engl J Med, 1989. 320(20): p. 1346-8.

29. Wiggs, B.R., C. Bosken, P.D. Pare, A. James, and J.C. Hogg. A model of airway narrowing in asthma and in chronic obstructive pulmonary disease. Am Rev Respir Dis, 1992. 145(6): p. 1251-8.

30. Berrizbeitia, L.D., S. Tessler, I.J. Jacobowitz, P. Kaplan, L. Budzilowicz, and J.N. Cunningham. Effect of sternotomy and coronary bypass surgery on postoperative pulmonary mechanics. Comparison of internal mammary and saphenous vein bypass grafts. Chest, 1989. 96(4): p. 873-6.

31. Vaidya, R., T. Husain, and P.K. Ghosh. Spirometric changes after open mitral surgery. J Cardiovasc Surg (Torino), 1996. 37(3): p. 295-300.

32. Tenling, A., T. Hachenberg, H. Tyden, G. Wegenius, and G. Hedenstierna. Atelectasis and gas exchange after cardiac surgery. Anesthesiology, 1998. 89(2): p. 371-8.

33. Taggart, D.P. Respiratory dysfunction after cardiac surgery: effects of avoiding cardiopulmonary bypass and the use of bilateral internal mammary arteries. Eur $J$ Cardiothorac Surg, 2000. 18(1): p. 31-7.

34. Benjamin, J.J., P.N. Cascade, M. Rubenfire, W. Wajszczuk, and N.Z. Kerin. Left lower lobe atelectasis and consolidation following cardiac surgery: the effect of topical cooling on the phrenic nerve. Radiology, 1982. 142(1): p. 11-4.

35. Jenkins, S.C., S.A. Soutar, A. Forsyth, J.R. Keates, and J. Moxham. Lung function after coronary artery surgery using the internal mammary artery and the saphenous vein. Thorax, 1989. 44(3): p. 209-11. 
36. Boldt, J., B. von Bormann, D. Kling, H.H. Scheld, and G. Hempelmann. The influence of extracorporeal circulation on extravascular lung water in coronary surgery patients. Thorac Cardiovasc Surg, 1986. 34(2): p. 110-5.

37. Gaar, K.A., Jr., A.E. Taylor, L.J. Owens, and A.C. Guyton. Pulmonary capillary pressure and filtration coefficient in the isolated perfused lung. Am J Physiol, 1967. 213(4): p. 910-4.

38. Petak, F., Z. Hantos, A. Adamicza, T. Asztalos, and P.D. Sly. Methacholine-induced bronchoconstriction in rats: effects of intravenous vs. aerosol delivery. J Appl Physiol, 1997. 82(5): p. 1479-87.

39. Hantos, Z., B. Daroczy, B. Suki, S. Nagy, and J.J. Fredberg. Input impedance and peripheral inhomogeneity of dog lungs. J Appl Physiol, 1992. 72(1): p. 168-78.

40. Fredberg, J.J. and D. Stamenovic. On the imperfect elasticity of lung tissue. J Appl Physiol, 1989. 67(6): p. 2408-19.

41. Petak, F., G.L. Hall, and P.D. Sly. Repeated measurements of airway and parenchymal mechanics in rats by using low-frequency oscillations. J Appl Physiol, 1998. 84(5): p. 1680-6.

42. Petak, F., Z. Hantos, A. Adamicza, H. Gality, and W. Habre. Development of bronchoconstriction after administration of muscle relaxants in rabbits with normal or hyperreactive airways. Anesth Analg, 2006. 103(1): p. 103-9, table of contents.

43. Miller, M.R., J. Hankinson, V. Brusasco, F. Burgos, R. Casaburi, A. Coates, R. Crapo, P. Enright, C.P. van der Grinten, P. Gustafsson, R. Jensen, D.C. Johnson, N. MacIntyre, R. McKay, D. Navajas, O.F. Pedersen, R. Pellegrino, G. Viegi, and J. Wanger. Standardisation of spirometry. Eur Respir J, 2005. 26(2): p. 319-38.

44. D'Angelo, E., M. Pecchiari, P. Baraggia, M. Saetta, E. Balestro, and J. Milic-Emili. Low-volume ventilation causes peripheral airway injury and increased airway resistance in normal rabbits. J Appl Physiol, 2002. 92(3): p. 949-56.

45. Habre, W., P. Scalfaro, N. Schutz, P. Stucki, and F. Petak. Measuring end-expiratory lung volume and pulmonary mechanics to detect early lung function impairment in rabbits. Respir Physiol Neurobiol, 2006. 152(1): p. 72-82.

46. Lutchen, K.R., Z. Hantos, F. Petak, A. Adamicza, and B. Suki. Airway inhomogeneities contribute to apparent lung tissue mechanics during constriction. $J$ Appl Physiol, 1996. 80(5): p. 1841-9.

47. Permutt, S., J.B. Howell, D.F. Proctor, and R.L. Riley. Effect of lung inflation on static pressure-volume characteristics of pulmonary vessels. J Appl Physiol, 1961. 16: p. 64-70.

48. Sakai, H., E.P. Ingenito, R. Mora, S. Abbay, F.S. Cavalcante, K.R. Lutchen, and B. Suki. Hysteresivity of the lung and tissue strip in the normal rat: effects of heterogeneities. J Appl Physiol, 2001. 91(2): p. 737-47.

49. Weibel, E.R., P. Untersee, J. Gil, and M. Zulauf. Morphometric estimation of pulmonary diffusion capacity. VI. Effect of varying positive pressure inflation of air spaces. Respir Physiol, 1973. 18(3): p. 285-308.

50. Smith, J.C. and W. Mitzner. Analysis of pulmonary vascular interdependence in excised dog lobes. J Appl Physiol, 1980. 48(3): p. 450-67.

51. Albu, G., W. Habre, F. Fontao, D.R. Morel, and F. Petak. The contribution of the pulmonary microvascular pressure in the maintenance of an open lung during mechanical ventilation. Respir Physiol Neurobiol, 2007. 157(2-3): p. 262-9.

52. Jasmin, J.F., A. Calderone, T.K. Leung, L. Villeneuve, and J. Dupuis. Lung structural remodeling and pulmonary hypertension after myocardial infarction: complete reversal with irbesartan. Cardiovasc Res, 2003. 58(3): p. 621-31. 
53. Kompa, A.R. and R.J. Summers. Lidocaine and surgical modification reduces mortality in a rat model of cardiac failure induced by coronary artery ligation. $J$ Pharmacol Toxicol Methods, 2000. 43(3): p. 199-203.

54. Uhlig, T., J.H. Wildhaber, N. Carroll, D.J. Turner, P.R. Gray, N. Dore, A.L. James, and P.D. Sly. Pulmonary vascular congestion selectively potentiates airway responsiveness in piglets. Am J Respir Crit Care Med, 2000. 161(4 Pt 1): p. 1306-13.

55. Wetzel, R.C., C.J. Herold, E.A. Zerhouni, and J.L. Robotham. Intravascular volume loading reversibly decreases airway cross-sectional area. Chest, 1993. 103(3): p. 86570 .

56. Hogg, J.C., J.B. Agarawal, A.J. Gardiner, W.H. Palmer, and P.T. Macklem. Distribution of airway resistance with developing pulmonary edema in dogs. J Appl Physiol, 1972. 32(1): p. 20-4.

57. Ishii, M., N. Matsumoto, T. Fuyuki, W. Hida, M. Ichinose, H. Inoue, and T. Takishima. Effects of hemodynamic edema formation on peripheral vs. central airway mechanics. J Appl Physiol, 1985. 59(5): p. 1578-84.

58. Noble, W.H., J.C. Kay, and J. Obdrzalek. Lung mechanics in hypervolemic pulmonary edema. J Appl Physiol, 1975. 38(4): p. 681-7.

59. Snashall, P.D. and K.F. Chung. Airway obstruction and bronchial hyperresponsiveness in left ventricular failure and mitral stenosis. Am Rev Respir Dis, 1991. 144(4): p. 94556.

60. Germonpre, P.R., G.F. Joos, and R.A. Pauwels. Modulation by 5-HT1A receptors of the 5-HT2 receptor-mediated tachykinin-induced contraction of the rat trachea in vitro. Br J Pharmacol, 1998. 123(8): p. 1571-8.

61. Lockhart, A., A.T. Dinh-Xuan, J. Regnard, L. Cabanes, and R. Matran. Effect of airway blood flow on airflow. Am Rev Respir Dis, 1992. 146(5 Pt 2): p. S19-23.

62. Light, R.W. and R.B. George. Serial pulmonary function in patients with acute heart failure. Arch Intern Med, 1983. 143(3): p. 429-33.

63. Schultz, H.D., T.E. Pisarri, H.M. Coleridge, and J.C. Coleridge. Carotid sinus baroreceptors modulate tracheal smooth muscle tension in dogs. Circ Res, 1987. 60(3): p. 337-45.

64. Pellegrino, R., R. Dellaca, P.T. Macklem, A. Aliverti, S. Bertini, P. Lotti, P. Agostoni, A. Locatelli, and V. Brusasco. Effects of rapid saline infusion on lung mechanics and airway responsiveness in humans. J Appl Physiol, 2003. 95(2): p. 728-34.

65. Baouz, S., J. Giron-Michel, B. Azzarone, M. Giuliani, F. Cagnoni, S. Olsson, R. Testi, G. Gabbiani, and G.W. Canonica. Lung myofibroblasts as targets of salmeterol and fluticasone propionate: inhibition of alpha-SMA and NF-kappaB. Int Immunol, 2005. 17(11): p. 1473-81.

66. Kapanci, Y., S. Burgan, G.G. Pietra, B. Conne, and G. Gabbiani. Modulation of actin isoform expression in alveolar myofibroblasts (contractile interstitial cells) during pulmonary hypertension. Am J Pathol, 1990. 136(4): p. 881-9.

67. Coleridge, J.C. and H.M. Coleridge. Afferent C-fibers and cardiorespiratory chemoreflexes. Am Rev Respir Dis, 1977. 115(6 Pt 2): p. 251-60.

68. Roberts, A.M., J. Bhattacharya, H.D. Schultz, H.M. Coleridge, and J.C. Coleridge. Stimulation of pulmonary vagal afferent C-fibers by lung edema in dogs. Circ Res, 1986. 58(4): p. 512-22.

69. Russell, J.A. and S.J. Lai-Fook. Reflex bronchoconstriction induced by capsaicin in the dog. J Appl Physiol, 1979. 47(5): p. 961-7.

70. Christian, E.P., G.E. Taylor, and D. Weinreich. Serotonin increases excitability of rabbit C-fiber neurons by two distinct mechanisms. J Appl Physiol, 1989. 67(2): p. 584-91. 
71. Chuaychoo, B., M.G. Lee, M. Kollarik, and B.J. Undem. Effect of 5hydroxytryptamine on vagal C-fiber subtypes in guinea pig lungs. Pulm Pharmacol Ther, 2005. 18(4): p. 269-76.

72. Ishaaya, A.M., S.D. Nathan, and M.J. Belman. Work of breathing after extubation. Chest, 1995. 107(1): p. 204-9.

73. Martin, S.E., R. Mathur, I. Marshall, and N.J. Douglas. The effect of age, sex, obesity and posture on upper airway size. Eur Respir J, 1997. 10(9): p. 2087-90.

74. Verheij, J., A. van Lingen, P.G. Raijmakers, J.J. Spijkstra, A.R. Girbes, E.K. Jansen, F.G. van den Berg, and A.B. Groeneveld. Pulmonary abnormalities after cardiac surgery are better explained by atelectasis than by increased permeability oedema. Acta Anaesthesiol Scand, 2005. 49(9): p. 1302-10.

75. Babik, B., T. Asztalos, F. Petak, Z.I. Deak, and Z. Hantos. Changes in respiratory mechanics during cardiac surgery. Anesth Analg, 2003. 96(5): p. 1280-7, table of contents.

76. Hachenberg, T., A. Tenling, H.U. Rothen, S.O. Nystrom, H. Tyden, and G. Hedenstierna. Thoracic intravascular and extravascular fluid volumes in cardiac surgical patients. Anesthesiology, 1993. 79(5): p. 976-84.

77. Kirklin, J.K., S. Westaby, E.H. Blackstone, J.W. Kirklin, D.E. Chenoweth, and A.D. Pacifico. Complement and the damaging effects of cardiopulmonary bypass. $J$ Thorac Cardiovasc Surg, 1983. 86(6): p. 845-57.

78. Magnusson, L., V. Zemgulis, S. Wicky, H. Tyden, S. Thelin, and G. Hedenstierna. Atelectasis is a major cause of hypoxemia and shunt after cardiopulmonary bypass: an experimental study. Anesthesiology, 1997. 87(5): p. 1153-63.

79. Friedrich, B., R. Schmidt, I. Reiss, A. Gunther, W. Seeger, M. Muller, J. Thul, D. Schranz, and L. Gortner. Changes in biochemical and biophysical surfactant properties with cardiopulmonary bypass in children. Crit Care Med, 2003. 31(1): p. 284-90.

80. Royston, D., B.D. Minty, T.W. Higenbottam, J. Wallwork, and G.J. Jones. The effect of surgery with cardiopulmonary bypass on alveolar-capillary barrier function in human beings. Ann Thorac Surg, 1985. 40(2): p. 139-43.

81. Barnas, G.M., R.J. Watson, M.D. Green, A.J. Sequeira, T.B. Gilbert, J. Kent, and E. Villamater. Lung and chest wall mechanical properties before and after cardiac surgery with cardiopulmonary bypass. J Appl Physiol, 1994. 76(1): p. 166-75.

82. Zupancich, E., D. Paparella, F. Turani, C. Munch, A. Rossi, S. Massaccesi, and V.M. Ranieri. Mechanical ventilation affects inflammatory mediators in patients undergoing cardiopulmonary bypass for cardiac surgery: a randomized clinical trial. J Thorac Cardiovasc Surg, 2005. 130(2): p. 378-83.

83. Jonmarker, C., L. Nordstrom, and O. Werner. Changes in functional residual capacity during cardiac surgery. Br J Anaesth, 1986. 58(4): p. 428-32.

84. Eichenberger, A., S. Proietti, S. Wicky, P. Frascarolo, M. Suter, D.R. Spahn, and L. Magnusson. Morbid obesity and postoperative pulmonary atelectasis: an underestimated problem. Anesth Analg, 2002. 95(6): p. 1788-92, table of contents.

85. Wright, J.R. Clearance and recycling of pulmonary surfactant. Am J Physiol, 1990. 259(2 Pt 1): p. L1-12.

86. Roosens, C., J. Heerman, F. De Somer, F. Caes, Y. Van Belleghem, and J.I. Poelaert. Effects of off-pump coronary surgery on the mechanics of the respiratory system, lung, and chest wall: Comparison with extracorporeal circulation. Crit Care Med, 2002. 30(11): p. 2430-7.

87. Kochamba, G.S., K.L. Yun, T.A. Pfeffer, C.F. Sintek, and S. Khonsari. Pulmonary abnormalities after coronary arterial bypass grafting operation: cardiopulmonary bypass versus mechanical stabilization. Ann Thorac Surg, 2000. 69(5): p. 1466-70. 